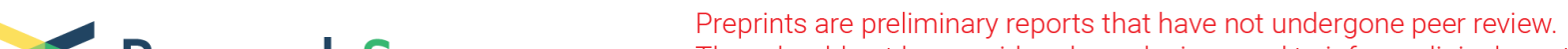 Research Square
They should not be considered conclusive, used to inform clinical practice,
or referenced by the media as validated information.
}

\section{Nanoscale Imaging of Phonon Dynamics by Electron Microscopy}

\section{Xiaoqing Pan ( $\nabla$ xiaoqinp@uci.edu )}

University of California Irvine https://orcid.org/0000-0002-0965-8568

\section{Chaitanya Gadre}

University of California, Irvine

\section{Xingxu Yan}

University of California Irvine https://orcid.org/0000-0001-7991-4849

\section{Qichen Song}

Massachusetts Institute of Technology https://orcid.org/0000-0002-1090-4068

Jie Li

University of California Irvine

Lei Gu

University of California, Irvine

\section{Toshihiro Aoki}

Arizona State University https://orcid.org/0000-0001-6620-9390

\section{Sheng-Wei Lee}

National Central University

\section{Gang Chen}

Massachusetts Institute of Technology https://orcid.org/0000-0002-3968-8530

\section{Ruqian Wu}

University of California, Irvine https://orcid.org/0000-0002-6156-7874

\section{Physical Sciences - Article}

Keywords: nanoscale imaging, microscopy, phonon dynamics

Posted Date: May 25th, 2021

DOl: https://doi.org/10.21203/rs.3.rs-540715/v1

License: (9) This work is licensed under a Creative Commons Attribution 4.0 International License. Read Full License 
Version of Record: A version of this preprint was published at Nature on June 8th, 2022. See the published version at https://doi.org/10.1038/s41586-022-04736-8. 


\section{Title: Nanoscale Imaging of Phonon Dynamics by Electron Microscopy}

Authors: Chaitanya A. Gadre ${ }^{1} \uparrow$, Xingxu Yan ${ }^{2,4} \uparrow$, Qichen Song ${ }^{3}$, Jie Li ${ }^{1}$, Lei Gu ${ }^{1}$, Toshihiro Aoki $^{4}$, Sheng-Wei Lee ${ }^{5}$, Gang $\mathrm{Chen}^{3}$, Ruqian Wu ${ }^{1}$, Xiaoqing Pan ${ }^{1,2,4 *}$

\section{Affiliations:}

${ }^{1}$ Department of Physics and Astronomy, University of California Irvine, CA 92697, United States

${ }^{2}$ Department of Materials Science and Engineering, University of California Irvine, CA 92697 , United States

${ }^{3}$ Department of Mechanical Engineering, Massachusetts Institute of Technology,

Cambridge, MA 02139, United States

${ }^{4}$ Irvine Materials Research Institute, University of California Irvine, CA 92697, United States

${ }^{5}$ Institute of Materials Science and Engineering, National Central University, Taoyuan, 32001 Taiwan

*Corresponding Author: xiaoqing.pan@uci.edu (X. P).

Keywords: Phonons, Vibrational Spectroscopy, Quantum Dots, SiGe superlattice, Principle Component Analysis, Phonon Reflection, non-equilibrium phonons

Spatially resolved vibrational mapping of nanostructures is indispensable to the development and understanding of thermal nanodevices ${ }^{1}$, modulation of thermal transport $^{2}$, and novel nanostructured thermoelectric materials ${ }^{3-5}$. Through the engineering of complex structures such as alloys, nanostructures, and superlattice interfaces, one can significantly alter the propagation of phonons and suppress material thermal conductivity while maintaining electrical conductivity ${ }^{2}$. Probing local vibrations and phonon dispersions in nanostructured semiconductors informs structure-property correlations and offers insights into the design and optimization of novel thermoelectric materials. There have been no correlative experiments that spatially track the modulation of phonon properties in and around nanostructures due to spatial resolution limitations of conventional optical phonon detection techniques. Here we demonstrate two-dimensional spatial mapping of phonons in a single silicon-germanium (SiGe) quantum dot (QD) using monochromated electron energy loss spectroscopy (EELS) in the transmission electron microscope (TEM). Tracking the variation of the $S i$ optical mode in and around the QD, we observe the nanoscale modification of the composition induced redshift. We observe nonequilibrium phonons that only exist near the interface and furthermore, develop a novel technique to 
differentially map phonon momenta providing direct evidence that the interplay between diffusive and specular reflection largely depends on the detailed atomistic structure --a major advancement in the field. Our work unveils the nonequilibrium phonon dynamics at nanoscale interfaces and can be used to study actual nanodevices and aid in the understanding of heat dissipation near nanoscale hotspots, which is crucial for future highperformance nanoelectronics. Our work demonstrates high spatial resolution vibrational characterization of nanostructures and interfaces that can be extended to other nanostructures and superlattice systems, in terms of composition, composition gradient, and structure driven phonon dynamics.

The control of phonon propagation and thermal conductivity of materials by nanoscale structural engineering is exceedingly important for the development and improvement of nanotransistors, thermal barriers, phase-change memory, and thermoelectric energy conversion ${ }^{1}$. For example, it has been a central issue to reduce the lattice thermal conductivity of thermoelectric materials for the enhancement of their figure of merit $(Z T)^{2,4-7}$. Although many single phase/crystal materials already possess high $Z T$ values due to the relatively high carrier mobility, their thermoelectric performance can be further enhanced by reducing thermal conductivity ${ }^{3,8,9}$. Numerous strategies have been employed to reduce the thermal conductivity or phonon transport of a given material by introducing material intermixing ${ }^{10}$, nanostructures ${ }^{2,4,6,9}$, and interfaces ${ }^{4,11}$. Through these mechanisms, short, medium, and long wavelength phonons are scattered, respectively. The SiGe QD superlattice structure is one such system that efficiently reduces thermal conductivity by 20 times by implementing all three phonon scattering mechanisms ${ }^{12}$.

Of these scattering mechanisms, interfacial phonon scattering is a subject of intensive study and has been largely carried out via modeling and simulation, combined with experimental measurement of the effective thermal conductivity of many layers ${ }^{13-15}$ rather than direct imaging of phonons. Although it is understood that phonon reflection at an interface is responsible for thermal boundary resistance ${ }^{16}$, there is no direct experimental observation of local phonon reflection. Raman spectroscopy has been used to study strain and compositional effects on phonons in $\mathrm{SiGe}$ superlattices ${ }^{17,18}$, while time-domain thermoreflectance measurements have been carried out to investigate thermal conductivity via ballistic transport ${ }^{19}$. However, both techniques lack spatial and momentum resolutions needed to study phonon dynamics of individual nanostructures and interfaces. Therefore, an experimental technique that probes nanoscale vibrational properties with high spatial, momentum, and energy resolutions is vital for deepening the understanding of nanoscale phonon transport physics.

Recent advances in monochromated electron microscopy have enabled the spectroscopy of vibrational excitations at the nanometer ${ }^{20}$ and even atomic ${ }^{21,22}$ scales. So far, 2D mapping of surface and bulk excitations ${ }^{23}$ and detection of single-atom ${ }^{24}$ and defect ${ }^{25}$ vibrational signals have been achieved. Although tdipole scattering in polar materials such as $\mathrm{BN}^{21,26,27}, \mathrm{MgO}^{23}$, and $\mathrm{SiC}^{20,28}$ produce long-range and non-local polariton modes posing a problem for high spatial resolution vibrational mapping, elemental and homopolar materials with weak dipoles such as $\mathrm{Si}^{22}$ and $\mathrm{SiGe}$ only contain high spatial resolution vibrational signatures. Here, we report quantitative high spatial resolution mapping of phonons in SiGe QDs using an on-axis beam-detector geometry 
(Extended data Fig. 1a). We experimentally reveal a remarkable phonon signal enhancement near the interface between $\mathrm{Si}$ and $\mathrm{SiGe} \mathrm{QD}$, which is confirmed to arise from the nanoscale phonon reflection near interfaces. This work serves as the foundation for future studies in nanoscale characterization of phonon propagation for the development and improvement of nanoscale structures and devices.
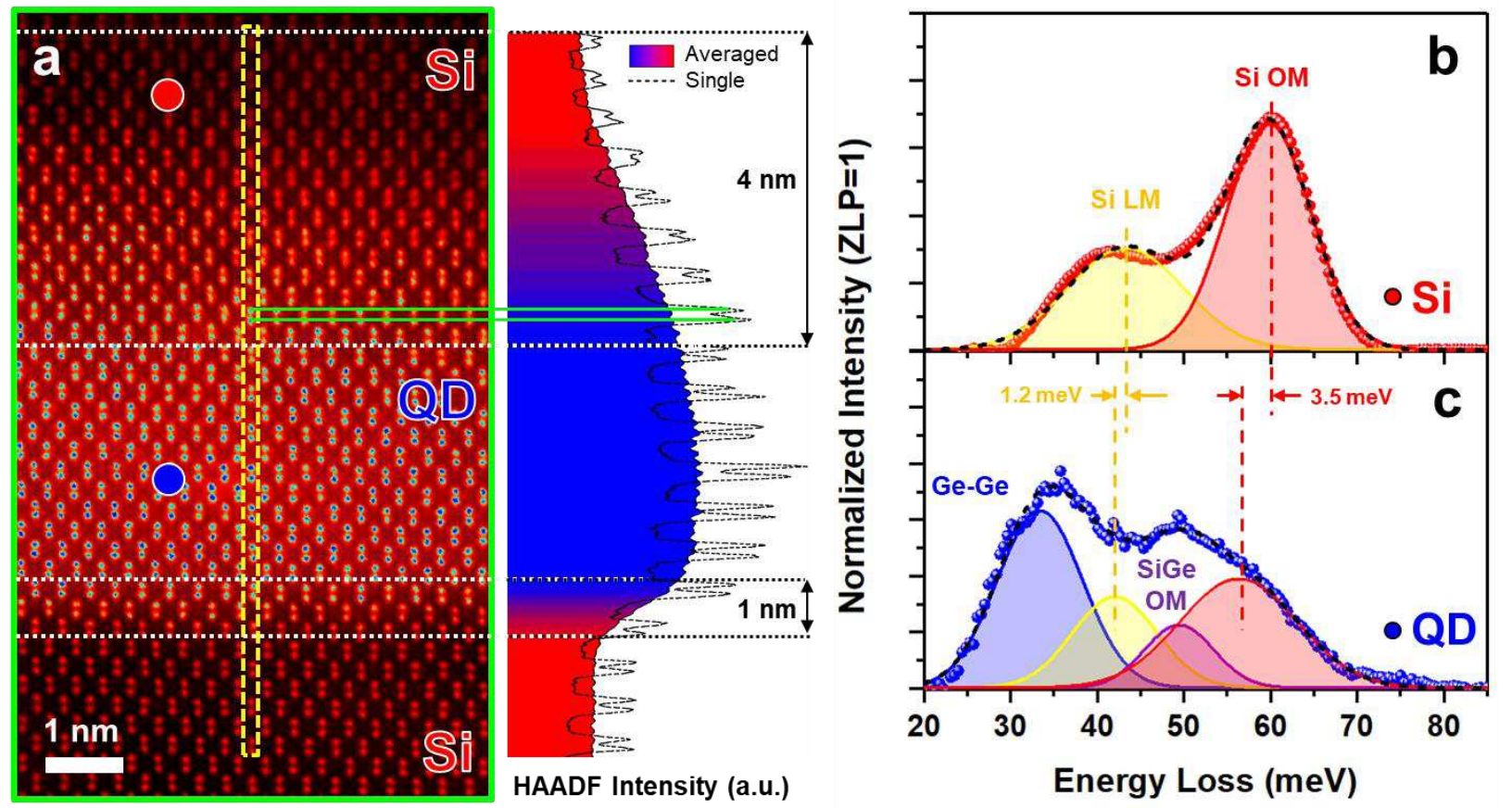

Fig. 1 Atomic structure and vibrational spectra of SiGe QD and Si-SiGe interfaces. a, Atomic resolution HAADF image containing both top and bottom QD interfaces from the region outlined in green in Extended Data Fig. 2. Line profile of a single array of atomic columns to the right is overlaid with a horizontally averaged profile of the entire image showing a gradual interface $4 \mathrm{~nm}$ wide and an abrupt one $1 \mathrm{~nm}$ wide for the top and bottom QD interfaces, respectively estimated by the $10-90 \%$ criterion. $\mathbf{b}, \mathbf{c}$, Background subtracted, pseudo-Voigt peak separated vibrational spectra of interlayer $\mathrm{Si}$ and SiGe QD from locations denoted by the red and blue dots in a, respectively.

The QDs chosen for this study were grown using the Stranski-Krastanov growth mechanism (more details in Methods) and those of similar dimensions were chosen for the study to exclude size variation effects (Extended Data Fig. 2a). Each QD has a dome-like interface at the top and a flat interface at the bottom (Extended Data Fig. 2b). These results are similar to previous results supported by atomic force microscopy and TEM characterizations ${ }^{29,30}$. Due to the Z-contrast property of high-angle annular dark field (HAADF) STEM imaging, the HAADF intensity can reflect the composition information of local regions. Utilizing this property, the widths of the top and bottom interfaces were measured to be about $4 \mathrm{~nm}$ and $1 \mathrm{~nm}$, respectively (Fig. 1a). The domelike interface at the top of the QD and flat interface at the bottom, closer to the substrate, are henceforth denoted as the gradual and abrupt interfaces, respectively. Elemental mapping results provide similar evidence of asymmetric Ge distribution inside the QDs as well and can be 
explained by Si diffusion from the top into the Ge layer during growth. Ge fractional composition, obtained by analyzing the core-loss EELS of Si $K$ edge and Ge $L$ edge (Extended Data Fig. 2c), varies almost monotonically with increasing layer number in the first several layers (Extended Data Fig. 2a) due to the growth conditions of the SiGe QD superlattice structure. Si undergoes thermal and strain activated diffusion into the SiGe QDs generating alloyed nanostructures ${ }^{31}$. As a result, $\mathrm{SiGe}$ QD layers closer to the substrate, which were grown first, experience higher amounts of Si diffusion than those that are farther away. This is the first time the nanoscale compositional variation is revealed in each layer for a SiGe QD superlattice structure as well as individual QDs. The varying composition of these QDs offers an interesting opportunity utilizing high resolution vibrational electron microscopy to investigate alloying effects on local vibration within a single sample, which is not possible for macroscopic optical methods ${ }^{18,32-34}$.

To study the compositional strain inside the QD, vibrational EEL spectra were acquired using an on-axis beam-detector geometry (Extended Data Fig. 1a). The acquired data was then processed by first subtracting the zero-energy loss background and then applying pseudo-Voigt peak decomposition to obtain properties of individual phonon modes (Figs. 1b and 1c) (More details in Materials and Methods, Extended Data Fig. 3). In the interlayer pure Si, two distinct Si-Si vibrational peaks are visible. The peak located at $59.8 \pm 0.2 \mathrm{meV}$ belongs to Si transverse and longitudinal optical (TO and LO) modes, denoted as OM, and another peak to the left which we classify as the low energy mode (LM) signal with an energy of $43.2 \pm 0.4 \mathrm{meV}$ (LA and optical phonon modes near zone edges). Inside the SiGe QD, there are 4 modes in the $20-80 \mathrm{meV}$ range corresponding to $\mathrm{Si}-\mathrm{Si}, \mathrm{Si}-\mathrm{Ge}$, and $\mathrm{Ge}-\mathrm{Ge}$ bonding vibrations with the $\mathrm{Si}-\mathrm{Si}$ vibration consisting of OM and LM (Fig. 1c). Starting from the highest energy mode, the Si OM has an energy of 56.3 $\pm 0.3 \mathrm{meV}$ followed by the Si-Ge optical vibration with an energy of $49.9 \pm 1.7 \mathrm{meV}$, Si LM with an energy of $42.0 \pm 1.2 \mathrm{meV}$, and lastly the Ge-Ge mode with an energy of $33.4 \pm 1.6 \mathrm{meV}$. Comparatively, the Si OM and LM energies being shifted in the SiGe QD by $3.5 \mathrm{meV}$ and 1.2 $\mathrm{meV}$, respectively. The Ge LM around $28 \mathrm{meV}$ was not separable as it largely overlapped with the Ge OM due to energy resolution limitations and was considered as a single Ge-Ge mode.

There is a $4 \mathrm{meV}$ discrepancy between the Raman and EELS Si OM energy in the pure Si region: $64.8 \mathrm{meV}$ with Raman (Extended Data Fig. 4) and $59.8 \mathrm{meV}$ with vibrational EELS. This discrepancy is accounted for by noting our experimental conditions (Extended Data Fig. 1). Since $33 \mathrm{mrad}$ and $25 \mathrm{mrad}$ convergence and collection semi-angles were used, electrons scattered at angles beyond even the second Brillouin zone (BZ) are included; a similar beam EELS configuration was used by K. Venkatraman et al. ${ }^{22}$ and can be considered a momentum averaged EELS acquisition geometry. While Raman spectroscopy only probes near-zero momentum phonons at the BZ center, vibrational EELS in our configuration probes phonons of all momenta thus producing the discrepancy between our results and those in Raman literature. The calculated phonon densities of states (Extended Data Figs. 1f and 1g) match well with Figs. 1b and 1c while also correctly capturing the momentum averaged Si OM peak position. Compared to Raman spectroscopy, the outstanding advantage of vibrational EELS is the superior spatial resolution, which reveals that the vibrational signal changes abruptly to within a nanometer when the probe is moved from the interlayer Si to the SiGe QD (Extended Data Fig. 5). 

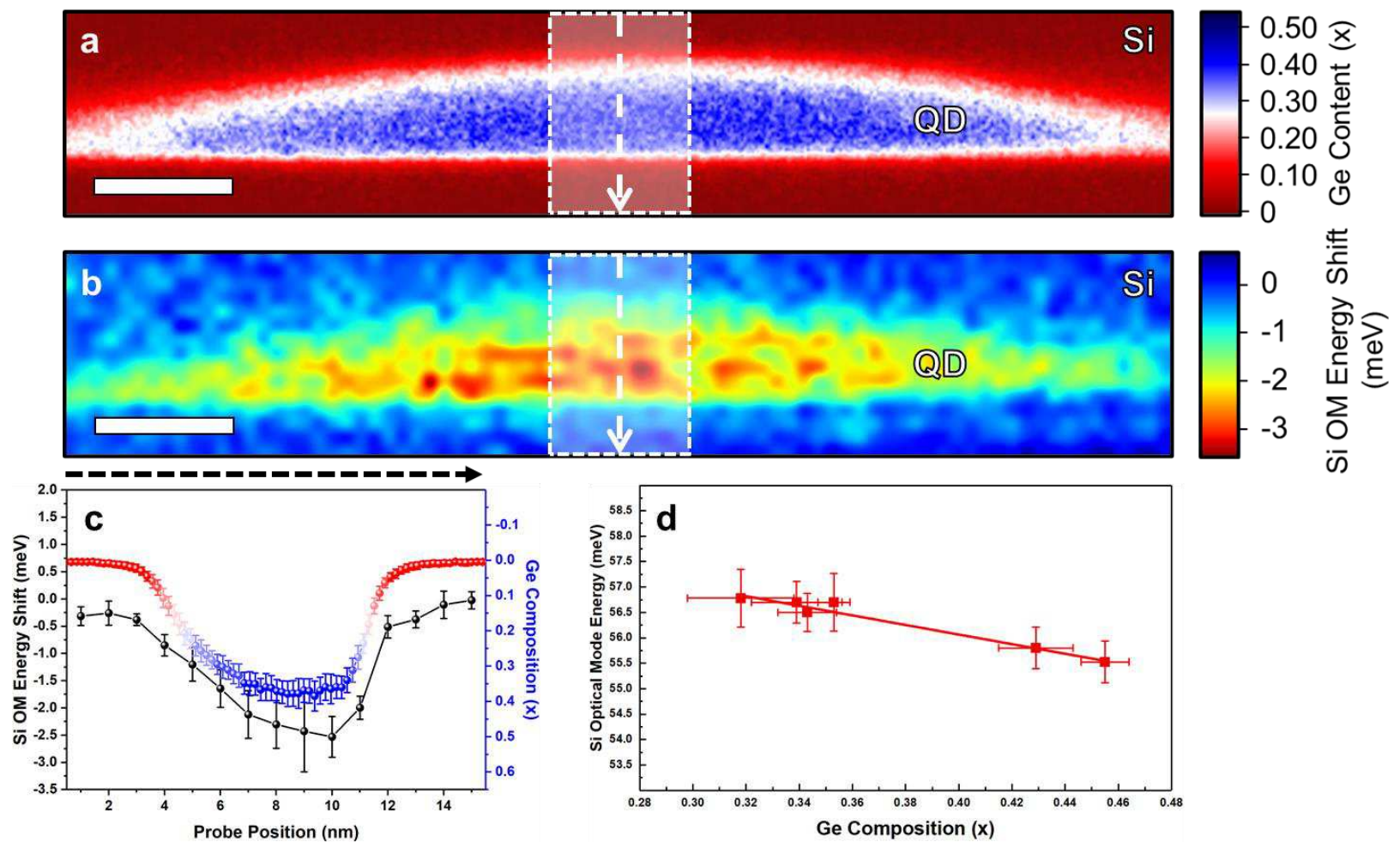

Fig. 2 | Spatial mapping of Ge concentration and Si OM energy shift in a single GD. a, Map of Ge composition acquired by core-loss EELS. The white contrast in the color map makes it easy to see relatively how far the interface extends around the QD. The red and blue colors indicate nearly 0 and 50 at. \% Ge concentration, respectively. b, Two-dimensional spatial mapping of Si OM energy of a single QD. The blue and ed color extremes denote standard and shifted energies, respectively. Scale bars in $\mathbf{a}$ and $\mathbf{b}$ are $10 \mathrm{~nm}$. c, A 10 horizontal-pixel-averaged 1D profile of Ge composition (colored) and Si OM energy (black) along the white dashed arrows in $\mathbf{a}$ and $\mathbf{b}$. d, Peak positions of Si OM in several QDs as germanium concentration $(x)$ in the center of the QD increases.

Vibrational EELS allows for the nanometer correlation of elemental and vibrational information (Figs. 2a and 2b). Both the composition and Si OM peak position maps were taken from the same QD. The $80 \times 15 \mathrm{~nm}$ dimensions of the Si OM energy shift map covers nearly the entire QD as well as surrounding interlayer Si and matches well with the QD shape confirming high spatial resolution. The Si OM energy shift is non-uniform inside the QD and has excellent tracking with the QD's Ge composition: Si OM energy shift is highest where the Ge content is highest (Fig. 2c). The asymmetry that is reflected in the $2 \mathrm{D}$ composition map is observed in the energy shift map and consistent with the observation in Fig. 1a. Meanwhile, we see that that bottom interface of the QD is much more abrupt than dome interface at the top, consistent with the aforementioned diffusion mechanism of the superlattice structure.

Furthermore, effects of varying composition across several QDs were investigated in a single sample effectively limiting the number of free variables in our experimental setup. Figure $2 \mathrm{~d}$ shows the energy shift trend of the Si OM as a function of the Ge composition $(x)$ at the center of 
corresponding QD's. Our data expresses a linear trend with a slope of $-9.3 \pm 1.09 \mathrm{meV} / x$ lying within accepted values in literature with values obtained by Raman spectroscopy ranging from 7.7 to $-8.8 \mathrm{meV} / x^{18,32-34}$ and a $y$-intercept of $59.8 \pm 0.4 \mathrm{meV}$ matching well with the momentum averaged $\mathrm{Si} \mathrm{OM}$ energy in pure $\mathrm{Si}$.
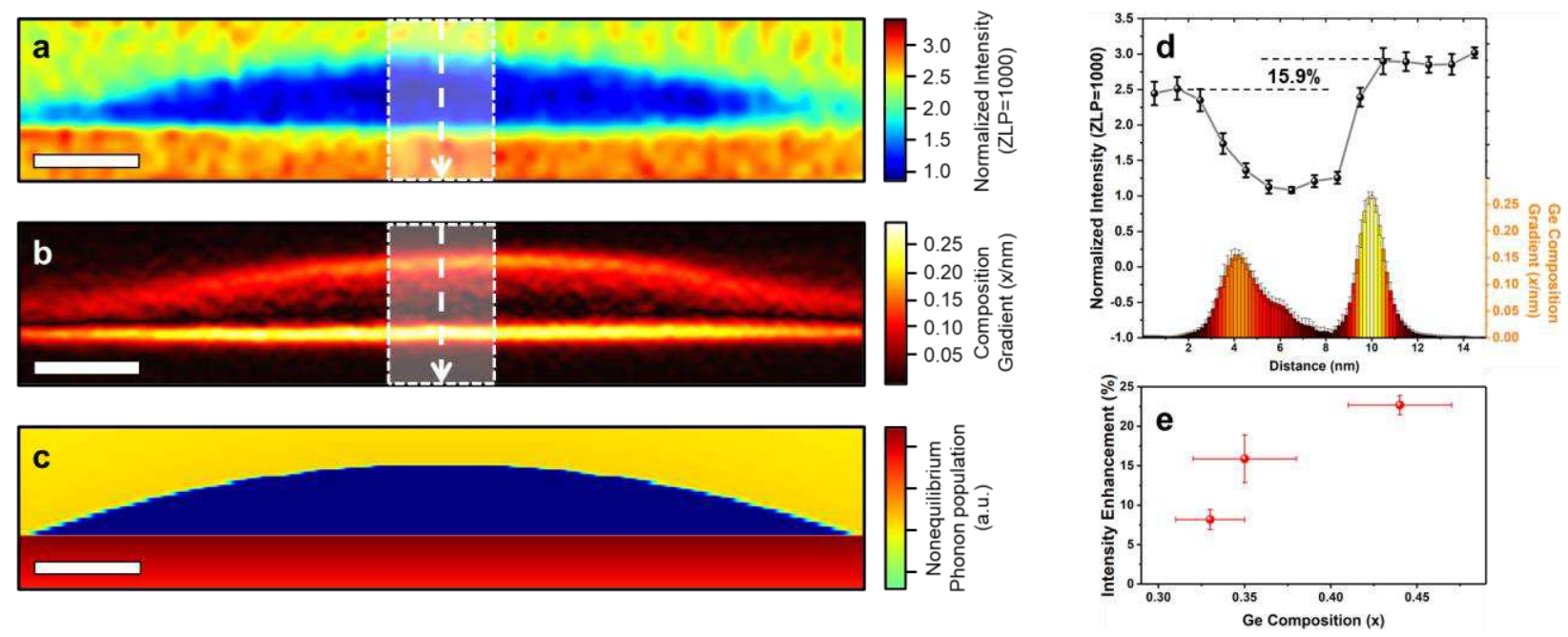

Fig. 3 | Asymmetric spectral intensities of Si OM near the gradual and abrupt interfaces. a, Two-dimensional spatial map of Si OM intensity of a 35 at. \% average Ge composition QD. The red and blue contrast extremes denote high and low relative mode intensities. b, Absolute value of the vertical gradient of the elemental map in Fig. 2a, emphasizing relative interface abruptness. Scale bars in a-c are $10 \mathrm{~nm}$ while the dashed arrows show the direction of the 1D profiles. c, Boltzmann transport simulated mapping of phonon population deviation from equilibrium of $\mathrm{Si}$ OM. Simulation was carried out only in Si side and only normal incident phonons are considered as phonons with oblique incidence do not contribute to the enhancement of EELS intensity. d, 1D profiles of the maps in $\mathbf{a}$ and $\mathbf{b}$. The 1D intensity line profile of $\mathbf{a}$ is plotted with the histogram profile of b. e Plot of Si OM intensity enhancement as a function of maximal Ge composition in the QD.

Figure 3a displays a 2D intensity mapping of the Si OM obtained from the same QD as in Fig. $2 \mathrm{~b}$. A striking feature is that the $\mathrm{Si} \mathrm{OM}$ at the bottom interlayer Si has a $15.9 \%$ intensity enhancement relative to that at the top one, highlighted in Fig. 3d, despite there being no compositional variance in the interlayer $\mathrm{Si}$ as evidenced by Fig. 2a. We posit that the source of this enhancement arises from the distinct scattering dynamics due to the two differing interfaces: an abrupt bottom interface and a gradual top interface (Figs. 3c and 3d). The momentum averaged scattering cross section is given by $\sigma=\int \sigma(\omega, \boldsymbol{q}) \mathrm{d} \boldsymbol{q}$ and the momentum-resolved Stokes scattering probability of fast electrons due to the lattice vibration near an interface has been derived ${ }^{26}$. To explain the experimental intensity enhancement, the local variations of phonon population $n$ and local density of states (LDOS) were considered individually. The computed LDOS showed no intensity enhancement likely due to the unchanged composition (Extended Data Fig. 6). Hence, the local non-equilibrium phonon occupation $n_{\boldsymbol{q}}$ is determined to be a significant factor for producing the intensity enhancement below the abrupt interface in Fig. 3a (more details in Supplementary Text). The 
nonequilibrium phonon occupation consists of two parts $n_{q v}=n_{q v, 0}+g_{q v}$, with $n_{q v, 0}$ being the equilibrium Bose-Einstein distribution at room temperature and $g_{q v}$ being the population deviation from equilibrium due to electron energy loss. Near the interface, optical phonons in Si side are reflected by the interface due to mode mismatch. Thus, the population deviation $g$ (the subscript is omitted) can be further decomposed into two parts $g=g_{0}+g^{\prime}$, the phonons emanating from the electron beam $g_{0}$ and the reflected phonons $g^{\prime}$. We use Boltzmann transport equation to solve for population deviation $g$ and find that

$g=g_{0}+A \cdot S \cdot R \exp (-z / \Lambda)$,

where $g_{0}$ and $A$ are constants that do not depend on interface properties, $z$ is the distance to interface, $\Lambda$ is the phonon mean free path in $\mathrm{Si}, S$ is the specularity parameter and $R$ is the reflectance of the interface. From atomic Green's function calculation, we find minimal difference in the reflectance for an abrupt interface and a gradual interface as a function of nanostructure curavture (Extended Data Fig. 9). Thus, we conclude that the difference in the nonequilibrium phonon population must be attributed to the difference in $S$ of the abrupt interface and the gradual interface. Our BTE simulation shows that the EELS intensity enhancement is attributed to higher $S$ of the abrupt interface than gradual interface. As the Ge content in the QD is increased, the composition change at the abrupt interface becomes more drastic leading to an intensity enhancement that exhibits a monotonic relationship with increasing Ge composition inside the QD, as confirmed by experimental data in Fig. 3e. Furthermore, the enhancement fades as phonons at the end of the QD are probed where the gradual and abrupt interfaces are less pronounced and nearly identical (Extended Data Fig. 11) because the thinner interface may have a smaller reflectance thus reducing the reflected phonon population equally on either side of the interface.

The reflection-induced nonequilibrium phonon population $g$ decays as a function of distance from the interface with the decay length being the mean free path (MFP) of the Si OM (Extended Data Fig. 12). A $20 \times 15 \mathrm{~nm}$ mapping of the interlayer Si bounded by two QDs illustrates a gradual change in the Si OM intensity (Extended Data Fig. 13). The top of the region is bounded by an abrupt interface while the bottom is bounded by a gradual interface. With the consideration that both interfaces have some degree of specularity, the data was fit with the sum of the two exponential decays arising from both the interfaces. With the fit, we obtained a MFP value of 25.1 $\mathrm{nm}$ for the $\mathrm{Si} \mathrm{OM}$ which is consistent with accepted values being in the range of tens of nanometers ${ }^{35}$, demonstrating that, for the first time, MFP of phonons can be measured at nanometer resolution.

The spot-like features in Fig. 3a and Extended Data Figs. 13 and 14 presumably arise from the short-range coulomb interaction between the beam and the atomic nucleus which is stronger when the beam is placed on the atomic column than when it is off, giving it a higher probability to excite a vibrational mode ${ }^{22}$. Interestingly, even when the maximum peak heights ( $\mathrm{Si} \mathrm{OM}$ ) are normalized to 1, the Ge OM still shows a strong modulation (Extended Data Fig. 14). This implies that this vibrational EELS technique may be more sensitive to heavier elements and suggests that even subÅngström level resolution is possible. 

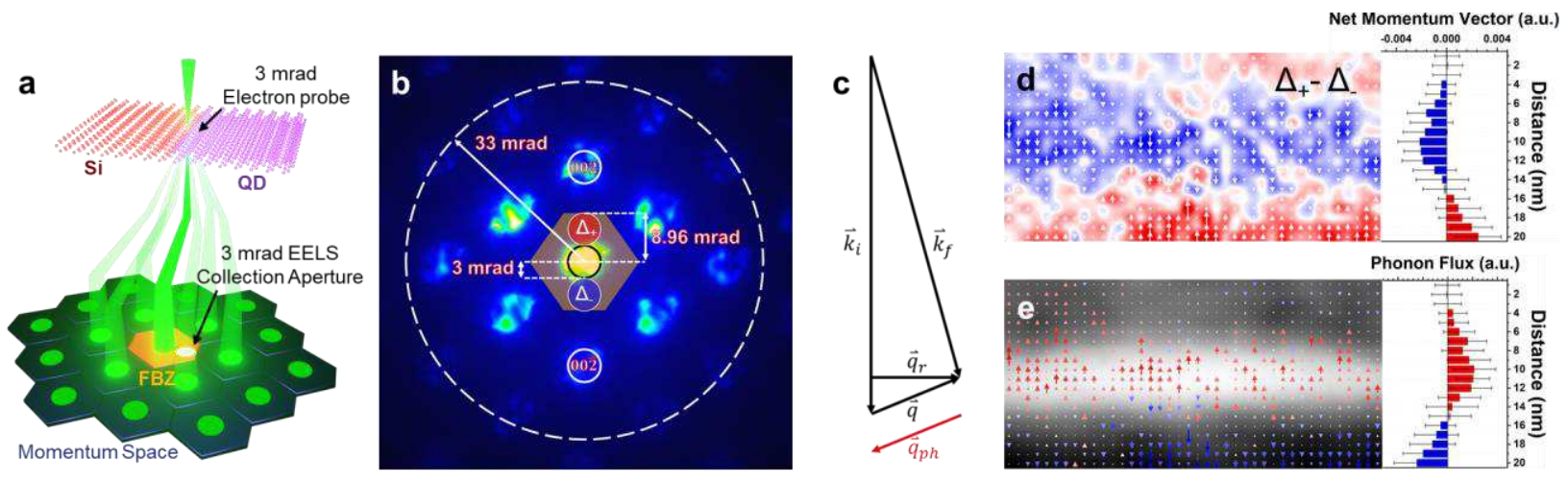

Fig. 4 | Momentum resolved differential mapping of phonon momentum normal to Gradual and Abrupt QD interfaces. a, Schematic of the experimental beam geometry showing an off-axis position of the EELS aperture in the Si FBZ achieved via post specimen tilting of the scattered electron beam. b, 3 mrad convergence semi-angle convergent beam electron diffraction pattern where the 002 and $00 \overline{2}$ spots are labeled. The orange hexagonal shape in the center denotes the first Brillion zone (FBZ) of Si with the $\Gamma-X$ distance labeled as $8.96 \mathrm{mrad}$. The red and blue circles near the center denote the EELS entrance aperture positions where off axis data was acquired. $\mathbf{c}$, Electron scattering wavevector diagram illustrating the fast electron-phonon momentum transfer. Under these momentum-resolved experimental conditions, $\vec{q} \approx \vec{q}_{r}$ meaning that momentum of generated phonons is predominantly in the in-plane direction and has an opposite sign to the momentum change of the electron beam. d, Mapping of net vertical momenta of Si optical phonons. e, Phonon flux vector map obtained from the product of group velocity and the quantity of phonons with a given momentum direction overlaid on a 3 mrad HAADF image. $\mathbf{d}$ and $\mathbf{e}$ are accompanied by their respective horizontally averaged line profiles.

To recover directional and momentum information and elucidate phonon dynamics at the QD interfaces, a momentum-resolved beam-detector geometry was employed (Fig. 4a). A convergence semi-angle of $3 \mathrm{mrad}$ (momentum resolution of $0.5 \AA^{-1}$ ) was used here which was about $1 / 3$ the size of the Si BZ as shown in Fig. 4b allowing for momentum resolution while the $33 \mathrm{mrad}$ area encompasses even neighboring BZ's (Extended Data Fig. 15a). Integrated spectral intensity in the $55-65 \mathrm{meV}$ region $(\mathrm{Si} \mathrm{OM})$ in the red and blue regions represents $\mathrm{Si}$ OM phonons created by electrons deflected towards the $002\left(\Delta_{+}\right)$and $00 \overline{2}\left(\Delta_{-}\right)$regions in reciprocal space, respectively (Extended Data Figs. 15b and 15c). Intriguingly, the intensity of the Si OM mode is higher in the

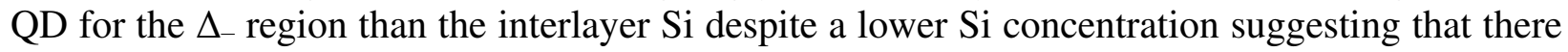
is a stronger preference for phonons to have a downward momentum in the QD. The same intensity enhancement as seen in Fig. 3a is recovered when intensity from summing both $\Delta_{+}$and $\Delta_{\text {- }}$ regions (Extended Data Fig. 15d).

Mapping the difference and considering momentum conservation (the phonon momentum vector is opposite to that of the electron momentum change direction as shown in Fig. 4c), a differential net phonon momentum map in the vertical direction is generated and phonon momenta directions are clearly seen experimentally at the nanoscale for the first time. For an atomically ideal interface, phonon reflection at interfaces is considered to be specular where the momentum parallel to the interface $q_{/ /}$is conserved due to the translational symmetry as seen. Conversely, for an atomically irregular interface, the atomic disorder breaks the transverse translational symmetry and consequently, phonon modes with different $q / /$ can also be coupled leading to a diffuse scattering 
process. The differential net momentum mapping in Fig. $4 \mathrm{~d}$ shows momentum vectors pointing towards the abrupt interface and given that optical phonons in Si have a group velocity in the opposite direction of their momentum from $\Gamma-X$ (Extended Data Fig. 1), Si optical phonons are then taken to be propagating away from the abrupt interface. The phonon flux, the product of group velocity and quantity of phonons with a given momentum shown in Fig. 4e, experimentally confirms this phenomenon and is consistent with the physics encapsulated by Figs. 3a and 3b. The more abrupt interface has a higher degree of specularity and therefore the generation of backward moving phonons are preferred while the more gradual interface at the top has much weaker directional preference due to its more gradual transition from Si to SiGe.

The ability to spatially map vibrations in nanostructured semiconductors is of paramount importance in the field of energy conversion, quantum computing, and nanotechnology in general. We have demonstrated that vibrational EELS is capable of probing interaction dynamics that manifest as modulations in the local population of vibrational states. Our results offer insight into nanoscale phonon physics at interfaces and experimentally show, for the first time, modulations in local vibrational states in the presence of nanostructures and interfaces. We have also obtained direct experimental evidence of dynamic phonon processes in the form of phonon reflection from interfaces at the nanoscale, unveiling the interplay between phonons and interface specularity. In conclusion, it is optimal for nanostructures to have an abrupt change in structure to achieve high phonon impedance thus lowering thermal conductivity. Beyond thermal transport, combining sub nanometer resolution with vibrational information offers an unprecedented level of access into nanoscale thermodynamics such as heat capacity and entropy.

\section{References and Notes:}

1. Cahill, D. G. et al. Nanoscale thermal transport. II. 2003-2012. Appl. Phys. Rev. 1, (2014).

2. Biswas, K. et al. High-performance bulk thermoelectrics with all-scale hierarchical architectures. Nature 489, 414-418 (2012).

3. Pei, Y. et al. Convergence of electronic bands for high performance bulk thermoelectrics. Nature 473, 66-69 (2011).

4. Pernot, G. et al. Precise control of thermal conductivity at the nanoscale through individual phonon-scattering barriers. Nat. Mater. 9, 491-495 (2010).

5. He, J. \& Tritt, T. M. Advances in thermoelectric materials research: Looking back and moving forward. Science (80-. ). 357, (2017).

6. Poudel, B. et al. High-thermoelectric performance of nanostructured bismuth antimony telluride bulk alloys. Science (80-. ). 320, 634-638 (2008).

7. Hochbaum, A. I. et al. Enhanced thermoelectric performance of rough silicon nanowires. Nature 451, 163-167 (2008).

8. Kim, W. et al. Thermal conductivity reduction and thermoelectric figure of merit increase by embedding nanoparticles in crystalline semiconductors. Phys. Rev. Lett. 96, 1-4 (2006). 
9. Harman, T. C., Harman, T. C., Taylor, P. J., Walsh, M. P. \& Laforge, B. E. Quantum Dot Superlattice Thermoelectric Materials and Devices. Science (80-. ). 2229, 2229-2232 (2008).

10. Rosi, F. D., Abeles, B. \& Jensen, R. V. Materials for thermoelectric refrigeration. J. Phys. Chem. Solids 10, 191-200 (1959).

11. Chen, G. Thermal conductivity and ballistic-phonon transport in the cross-plane direction of superlattices. Phys. Rev. B 57, 958-973 (1998).

12. Liu, W. L., Borca-Tasciuc, T., Chen, G., Liu, J. L. \& Wang, K. L. Anisotropic Thermal Conductivity of Ge Quantum-Dot and Symmetrically Strained Si/Ge Superlattices. J. Nanosci. Nanotechnol. 1, 39-42 (2001).

13. Lee, S.-M., Cahill, D. G. \& Venkatasubramianian, R. Thermal conductivity of Si and Ge. Appl. Phys. Lett. 77, 2957-2959 (1997).

14. Luckyanova, M. N. et al. Coherent Phonon Heat Conduction in Superlattices. Science (80. ). 338, 936-939 (2012).

15. Ravichandran, J. et al. Crossover from incoherent to coherent phonon scattering in epitaxial oxide superlattices. Nat. Mater. 13, 168-172 (2014).

16. Swartz, E. T. \& Pohl, R. O. Thermal boundary resistance. Rev. Mod. Phys. 61, 605-668 (1989).

17. Liu, J. L. et al. Optical and acoustic phonon modes in self-organized Ge quantum dot superlattices. Appl. Phys. Lett. 76, 586-588 (2000).

18. Groenen, J. et al. Phonons as probes in self-organized SiGe islands. Appl. Phys. Lett. 71, 3856-3858 (1997).

19. Hu, Y., Zeng, L., Minnich, A. J., Dresselhaus, M. S. \& Chen, G. Spectral mapping of thermal conductivity through nanoscale ballistic transport. Nat. Nanotechnol. 10, 701-706 (2015).

20. Krivanek, O. L. et al. Vibrational spectroscopy in the electron microscope. Nature 514, 209-212 (2014).

21. Hage, F. S., Kepaptsoglou, D. M., Ramasse, Q. M. \& Allen, L. J. Phonon Spectroscopy at Atomic Resolution. Phys. Rev. Lett. 122, 16103 (2019).

22. Venkatraman, K., Levin, B. D. A., March, K., Rez, P. \& Crozier, P. A. Vibrational spectroscopy at atomic resolution with electron impact scattering. Nat. Phys. 15, 12371241 (2019).

23. Lagos, M. J., Trügler, A., Hohenester, U. \& Batson, P. E. Mapping vibrational surface and bulk modes in a single nanocube. Nature 543, 529-532 (2017). 
24. Hage, F. S., Radtke, G., Kepaptsoglou, D. M., Lazzeri, M. \& Ramasse, Q. M. Single-atom vibrational spectroscopy in the scanning transmission electron microscope. Science (80-. ). 367, 1124-1127 (2020).

25. Yan, X. et al. Single-defect phonons imaged by electron microscopy. Nature 589, 65-69 (2021).

26. Senga, R. et al. Position and momentum mapping of vibrations in graphene nanostructures. Nature 573, 247-250 (2019).

27. Idrobo, J. C. et al. Temperature Measurement by a Nanoscale Electron Probe Using Energy Gain and Loss Spectroscopy. Phys. Rev. Lett. 120, 95901 (2018).

28. Yan, X. et al. Unexpected Strong Thermally Induced Phonon Energy Shift for Mapping Local Temperature. Nano Lett. 19, 7494-7502 (2019).

29. Chang, H. T., Wang, S. Y. \& Lee, S. W. Designer Ge/Si composite quantum dots with enhanced thermoelectric properties. Nanoscale 6, 3593-3598 (2014).

30. Mateeva, E., Sutter, P., Bean, J. C. \& Lagally, M. G. Mechanism of organization of threedimensional islands in SiGe / Si multilayers. Appl. Phys. Lett. 3233, (1997).

31. Schmidt, O. \& Eberl, K. Multiple layers of self-asssembled Ge/Si islands:

Photoluminescence, strain fields, material interdiffusion, and island formation. Phys. Rev. B - Condens. Matter Mater. Phys. 61, 13721-13729 (2000).

32. Alonso, M. I. \& Winer, K. Raman spectra of c-Si1-xGex alloys. Phys. Rev. B 39, 1005610062 (1989).

33. Tsang, J. C., Mooney, P. M., Dacol, F. \& Chu, J. O. Measurements of alloy composition and strain in thin $\mathrm{Ge}_{x} \mathrm{Si}_{1-x}$ layers. J. Appl. Phys. 75, 8098-8108 (1994).

34. Nakashima, S., Mitani, T., Ninomiya, M. \& Matsumoto, K. Raman investigation of strain in $\mathrm{Si} / \mathrm{SiGe}$ heterostructures: Precise determination of the strain-shift coefficient of Si bands. J. Appl. Phys. 99, 053512 (2006).

35. Liao, B. et al. Significant reduction of lattice thermal conductivity by the electron-phonon interaction in silicon with high carrier concentrations: A first-principles study. Phys. Rev. Lett. 114, 1-6 (2015).

36. Blöchl, P. E. Projector augmented-wave method. Phys. Rev. B 50, 17953-17979 (1994).

37. Kresse, G. \& Joubert, D. From ultrasoft pseudopotentials to the projector augmented-wave method. Phys. Rev. B - Condens. Matter Mater. Phys. 59, 1758-1775 (1999).

38. Perdew, J. P., Burke, K. \& Ernzerhof, M. Generalized gradient approximation made simple. Phys. Rev. Lett. 77, 3865-3868 (1996). 
39. Gonze, X. \& Lee, C. Dynamical matrices, Born effective charges, dielectric permittivity tensors, and interatomic force constants from density-functional perturbation theory. Phys. Rev. B - Condens. Matter Mater. Phys. 55, 10355-10368 (1997).

40. Chen, G. Nanoscale energy transport and conversion: a parallel treatment of electrons, molecules, phonons, and photons. Oxford Univ. Press (2005). doi:10.5860/choice.43-2818

41. Lambin, P. \& Vigneron, J. P. Computation of crystal Green's functions in the complexenergy plane with the use of the analytical tetrahedron method. Phys. Rev. B 29, 34303437 (1984).

42. Stillinger, F. H. \& Weber, T. A. Computer simulation of local order in condensed phases of silicon. Phys. Rev. B 31, 5262-5271 (1985).

43. Khomyakov, P. A., Brocks, G., Karpan, V., Zwierzycki, M. \& Kelly, P. J. Conductance calculations for quantum wires and interfaces: Mode matching and Green's functions. Phys. Rev. B - Condens. Matter Mater. Phys. 72, 1-13 (2005).

44. Giannozzi, P. et al. QUANTUM ESPRESSO: A modular and open-source software project for quantum simulations of materials. J. Phys. Condens. Matter 21, (2009).

45. Togo, A. \& Tanaka, I. First principles phonon calculations in materials science. Scr. Mater. 108, 1-5 (2015).

Acknowledgements: TEM and Raman experiments was conducted using the facilities in the Irvine Materials Research Institute (IMRI) and the Laser Spectroscopy Labs at the University of California-Irvine, respectively. The authors acknowledge Dr. Chris Meyer from Nion company for his support in the development of custom python acquisition code used to acquire data for this study and Dr. Ondrej L. Krivanek for his aid in preparing the manuscript. Funding: This work was primarily supported by the Department of Energy (DOE), Office of Basic Energy Sciences, Division of Materials Sciences and Engineering under Grant No. DE-SC0014430, and partially supported by the National Science Foundation Materials Research Science and Engineering Center program through the UC Irvine Center for Complex and Active Materials (DMR-2011967). DFT and BTE based simulations were supported by DOE Basic Energy Science under award number DE-FG02-05ER46237 (J.L., L.G. and R.W.) and by MRSEC Program of the National Science Foundation under award number DMR-1419807 (Q.S. and G.C.) and National Energy Research Scientific Computing Center (NERSC). Author contributions: C.A.G. and X.Y. contributed equally to this work. X.P. conceived and directed this project. C.A.G. designed the experiments. C.A.G. and X.Y. carried out the EELS experiments with the help of T.A. C.A.G. developed custom code to process the data and carried out the analysis for all STEM-EELS data. Standard phonon dispersion curve and density of states simulations were carried out by J.L., L.G. and R.W. BTE, Green's function, and DFT simulations were carried out by Q.S. and G.C. The SiGe QD superlattice structure was grown by S.W.L and the TEM sample was prepared by C.A.G. The manuscript was prepared by C.A.G., X.Y., Q.S., G.C., X.P. with input from all co-authors. Competing interests: The authors declare no competing interests. Data and materials availability: Data, materials, and code are available upon request. 


\section{Methods}

Fabrication of SiGe Quantum Dot Superlattice Sample and Preparation of TEM Sample

The quantum dot sample was grown at $600{ }^{\circ} \mathrm{C}$ in an ultra-high vacuum chemical vapor deposition (UHV/CVD) system. For $\mathrm{Ge}$ and $\mathrm{Si}$ depositions, pure $\mathrm{GeH}_{4}$ and $\mathrm{SiH}_{4}$ gases were used as precursors, respectively. The $\mathrm{Si}$ wafers were first etched in a diluted HF solution to create a hydrogen-passivated surface prior to deposition. After the deposition of a 50-nm-thick buffer layer of $\mathrm{Si}, \mathrm{Ge}$ layers were grown with $20 \mathrm{~nm} \mathrm{Si} \mathrm{spacer} \mathrm{layers} \mathrm{in} \mathrm{between} \mathrm{each} \mathrm{Ge} \mathrm{quantum} \mathrm{dot} \mathrm{layer}$ for the formation of self-assembled conventional QDs. For investigating thermal stability and tunability of the structural parameters, in-situ post deposition annealing was conducted at the growth temperature for 1 hour. The final product was a thin-film-like material of 40-period multifold QDs stacks with a thickness as high as $\sim 1.2 \mu \mathrm{m}$. QD nanostructures spanned 70-80 nm across and were 6-8 nm thick. More details of the growth process can be found in Ref. ${ }^{29}$. cross-sectional TEM sample used in this study was prepared by focused ion beam milling. The QD interfaces were normal to the (001) crystallographic plane while the zone axis was along the [110] crystallographic direction.

\section{$\underline{\text { Raman Spectroscopy }}$}

Raman spectra were acquired using a Renishaw inVia confocal Raman microscope. Point spectra in the QD sample and Si wafer were acquired in 50 1-second frames and then summed to achieve high signal-to-noise ratio.

\section{$\underline{\text { STEM Imaging }}$}

Images were taken with the Nion UltraSTEM 200 HERMES system operating at $60 \mathrm{kV}$ and 200 $\mathrm{kV}$ acceleration voltages with $33 \mathrm{mrad}$ and $34 \mathrm{mrad}$ convergence angles producing $1.5 \AA$ and 0.78 $\AA$ sized probes, respectively. The HAADF signal were collected by a high-angle annular detector with inner and outer collection angles of $103 \mathrm{mrad}$ and $210 \mathrm{mrad}$, respectively. Beam current was approximately $100 \mathrm{pA}$ in the imaging condition.

\section{Composition EELS Mapping}

2D elemental maps were acquired using the double Cs corrected $300 \mathrm{kV}$ JEOL Grand ARM $\mathrm{S} / \mathrm{TEM}$ system. A $0.5 \mathrm{eV} /$ pixel dispersion was used for elemental mapping with a convergence of $25 \mathrm{mrad}$ and a collection angle of $100 \mathrm{mrad}$. Spectra were acquired in the 500-2355 eV range including Ge $L$ edge at $1217 \mathrm{eV}$ and Si $K$ edge at $1839 \mathrm{eV}$ (Extended Data Fig. 2c). Single QD elemental maps were acquired with a $0.5 \mathrm{~nm} /$ pixel step size while low magnification spectra were acquired with $2 \mathrm{~nm}$ step size and were used to determine the composition distribution in different

QD layers. The core-loss signals of Si and Ge were background subtracted and integrated using Gatan's Digital Micrograph software. The histogram data reported in Extended Data Fig. 2a was obtained by averaging $4 \times 4$-pixel centers of each quantum dot.

\section{High Energy Resolution Monochromated EELS Experiments}


Experimental data was acquired on a Nion-UltraSTEM 200 microscope with the High Energy Resolution Monochromated EELS System (HERMES) operating at $60 \mathrm{kV}$ to achieve a balance between high spatial resolution and high inelastic scattering probabilities by electrons for vibrational spectroscopy. EEL spectra were produced by low angle scattered electrons (Extended Data Fig. 1a). Combining the monochromator with a high dispersion spectrometer, we achieved the best energy resolution of $5.7 \mathrm{meV}$ with the probe in vacuum for a $10 \mathrm{~ms}$ acquisition at $60 \mathrm{keV}$. A CMOS camera collects a 2D spectrum image which is then cropped and flattened in the undispersed direction to produce a 1D EELS spectrum.

\section{Momentum Averaged 33mrad Condition}

EELS acquisition was performed using a 33 mrad high current probes with resolution and beam current described in the STEM imaging section. A high probe current is necessary for attaining high signal-to-noise ratio spectra at the cost of slightly decreasing spatial resolution. Insertion of the monochromating slit reduces the probe current to about 3 pA but gives an energy width of 8.3 meV for an acquisition time of 1 second with the probe placed on the sample. This allows for accurately probing the optical mode of germanium. An EELS entrance aperture of $1 \mathrm{~mm}$ subtends a 25-mrad EELS collection semi-angle. With the Brillouin zone of silicon having a $\Gamma$-X length of only $8.96 \mathrm{mrad}$, a collection semi-angle of $25 \mathrm{mrad}$ collects inelastically scattered electrons from multiple neighboring Brillouin zones as well.

Spectral data was acquired in the form of line scans and 2D maps with the monochromating slit inserted. For line scans, 100 spectral frames were collected at 1 second exposure, aligned by their respective zero-loss peak (ZLP) maximums and then summed for each probe position. The dark current spectrum was frequently obtained during experiments. Aligning individual frames by ZLP averages for any random noise present in each frame. Maximizing single exposure time without saturating the EELS camera provided the best signal-to-noise ratio. Implementation of this acquisition scheme was achieved using Nion Swift software and custom python scripts designed to directly control necessary hardware parameters.

Phonon maps of an entire SiGe QD consist of nanometer-step $80 \times 15$ array of data points that were obtained with 1-1.5 second exposures and 5 frames per pixel. Due to the large size of the map, number of integrated frames were minimized to limit sample drift and drops in emission current overtime. Typical acquisition time for maps was about 2-3 hours and presented several challenges to acquiring high quality data: sample contamination, drift, gradual drop in beam current, and deterioration of energy resolution due to low order spectrometer aberrations. To mitigate these effects, the following precautions and procedures were undertaken:

1. The instrument was tuned to optimal EELS acquisition parameters achieving an energy resolution of about $8.3 \mathrm{meV}$ on the sample and was stabilized for at least 4 hours. The experiment was then performed the morning after and owing to the UltraSTEM's remarkable stability, a sample drift of less than $5 \AA$ /hour was present.

2. Custom mapping code allowed for the flashing of the tip every hour to ensure a high beam current necessary for optimal signal-to-noise ratio as well as the automatic correction of 
EELS first order aberrations every few points to maintain a high energy resolution throughout the experiment.

\section{Momentum Resolved 3mrad Condition:}

With a $3 \mathrm{mrad}$ convergence semi-angle and $2.5 \mathrm{mrad}$ collection semi-angle, the real-space probe size was estimated to be $2.6 \mathrm{~nm}$ and on-axis energy resolution to be $8.3 \mathrm{meV}$ in vacuum and 9.1 $\mathrm{meV}$ on the sample. Momentum differential mapping was carried out by collecting off-axis spectral intensity within the FBZ. Off-axis collection was achieved by using a combination of postspecimen lenses to shift the diffraction image while keeping the real-space probe stationary, so that the region of interest in reciprocal space lies upon the EELS entrance aperture (Extended Data Fig. 15). Energy resolution in this condition was estimated to be $12-14$ meV making peak separation difficult. Acquired maps were $30 \times 20$ in size with a step size of $1 \mathrm{~nm} /$ pixel and $5 \mathrm{~s}$ exposure. Sample was stabilized to minimize drift so that data from both areas symmetric about the $\Gamma$ could be acquired from the same QD with less than $1 \mathrm{~nm}$ of drift.

\section{$\underline{\text { Spectral Data Processing }}$}

All acquired spectra were processed using custom python codes. Spectra were first normalized with respect to the ZLP maximum so that each vibrational intensity represented a fractional scattering probability. Spectra were then background subtracted using a linear combination of a Lorentzian centered on the ZLP and an exponential polynomial $e^{\mathrm{p}(\mathrm{x})}$ where $\mathrm{p}(\mathrm{x})$ is an even $4^{\text {th }}$ order polynomial. This function was fitted to energy windows before and after the regions of interest. This combination produced a sharply decreasing left end and a slow and stable right tail necessary for accurately extracting the low energy optical modes of germanium. The fit was obtained using scipy.optimize, a python library, and fitting coefficients and covariances were extracted. To determine the efficacy of the background fit, error values obtained from the square root of the diagonal terms of the covariance matrix, were examined and minimized. The background subtracted signals were scrutinized for any negative, non-physical values.

By fitting the vibrational EELS signal with pseudo-Voight fits which are linear combinations of gaussians and lorentzians, we can gather information about a mode's excitation probability and its energy position given by the fitting intensity and peak position outputs. Individual inelastic probabilities were separated from background subtracted spectra by performing pseudo-Voight fits using curve_fit() from scipy.optimize. Fitting parameters of individual peaks and their corresponding errors were extracted. We examined the value of the errors obtained from the covariance of the fit to validate our peak separation. Due to the nature of the convoluted signal, phonon dispersion curves and Raman scattering data were used to accurately deconvolute the overlapping peaks. Lower bounds of 0 and upper bounds of infinity and $40 \mathrm{meV}$ were placed on the height and width of the pseudo-Voight fits, respectively. The energy position bounds were chosen to be $\pm 5 \mathrm{meV}$ from their initial reference position to avoid crossing of the separate pseudoVoight fits. 
Momentum resolved spectra were first trimmed and processed by binned principal component analysis (PCA) before background subtraction due to the low phonon scattering cross section under this beam geometry. After background subtraction, due to the poor energy resolution and low intensity, peak separation was not feasible, and a simple integration was carried out instead to obtain the Si optical mode intensity. The 2D maps were created by integrating the signal in the 55$65 \mathrm{meV}$ energy region in each pixel.

\section{Principle Component Analysis}

Given the acquisition parameters of the line scans, the excellent signal-to-noise ratio was adequate to accurately separate the individual modes. The low acquisition parameters for mapping datasets required the use of principle component analysis (PCA) to improve the signal quality. Rather than using Fourier filtering or other smoothening techniques, PCA learns from the large map dataset and reconstructs the spectra, maintaining important features while improving their quality.

Map data was first background subtracted and trimmed to reduce feature size. The dataset was then arranged as $N \times D$, where $N$ represents background subtracted spectra at different points and $D$, the pixel intensity of each individual signal. Three principal eigenvectors described most of the data and including additional eigenvectors offered only a fraction of a percent increase in cumulative explained variance. PCA was employed on map sizes of $80 \times 15$ producing a total of $N$ $=1200$ data points and trimmed background subtracted spectra of only $D=182$ pixels achieving an ideal condition for this machine learning algorithm to produce excellent results (Extended Data Fig. 17).

Off-axis momentum resolved data could not be smoothened the same way due to the smaller collection angle and modified phonon scattering cross section. Standard PCA fails to smoothen the data as the dominant feature is noise. Instead, we create a superset of the map data by binning the pixels and adding them to the original map dataset. Binning averages out the noise while maintaining the spectral features of interest. First, map data is binned by 2 then 3, etc each successively added to the original dataset to create a superset. With the addition of binned data back into the dataset, PCA functions as intended and accurately smoothens the data. The same criteria as above was employed in selecting the proper number of eigenvectors.

\section{Data Visualization}

Contour plots of mapping data were created using matplotlib.pyplot, another python library. Extracted parameters from the pseudo-Voigt fitting were then used to create a spatially resolved 2D map. Maps were generated using a gaussian interpolation for better visualization. Phonon mode intensity maps and peak position maps were constructed for silicon optical modes in units of normalized intensity and $\mathrm{meV}$, respectively.

Phonon Dispersion and Density of States simulations of bulk Si and Ge

Our first-principles calculations are carried out using the Vienna ab-initio simulation package (VASP) with the projector augmented wave (PAW) method is adopted for the interaction between valence electrons and ionic cores ${ }^{36,37}$, and the energy cutoff for the plane wave basis expansion 
was set to $700 \mathrm{eV}$. The generalized-gradient approximation (GGA) with the functional developed by Perdew-Burke-Ernzerhof (PBE) is chose for the exchange-correlation functional ${ }^{38}$. All atoms were fully relaxed using the conjugated gradient method for the energy minimization until the force on each atom became smaller than $0.01 \mathrm{eV} / \AA$. The phonon spectrum and the corresponding phonon density of states are obtained using density-functional perturbation theory ${ }^{39}$. 


\section{Supplementary Text}

The effective temperature rise caused by electron beam

A schematic depicting the generation of phonons by the electron beam is given in Extended Data Fig. 7. In the following, we will estimate how many phonons are generated by the electron beam per unit time. The electron beam current through the sample is measured to be $3 \mathrm{pA}$ meaning that there are 0.019 electrons travelling through sampler per ns. If we consider the beam as a stream of consecutive single electrons, the average time interval between electrons transmitting through sample is $53 \mathrm{~ns}$. By examining the EELS intensity, $I(E)$, we estimate the average energy loss per electron: $\Delta E=\int(\Delta E) I(E) \mathrm{d} E / \int I(E) \mathrm{d} E=12.5 \mathrm{eV}$, where $\Delta E$ denotes the energy loss of the fast electron, respectively (Extended Data Fig. 18). Although the energy loss is in various forms, for example plasmons, eventually the energy loss all become heat energy to excite phonons with various decay times. From the density of states computed using first-principles calculations, we find the average phonon energy $E_{p h}=\int \hbar \omega D(\omega) \mathrm{d} \omega / \int D(\omega) \mathrm{d} \omega=40.4 \mathrm{meV}$. Then, the number of $\mathrm{Si}$ OM phonons per electron is given by $n_{S i O M}=\int_{\omega_{1}}^{\omega_{2}} \hbar \omega D(\omega) \mathrm{d} \omega / \int D(\omega) \mathrm{d} \omega=105$ Si OM phonons where $\hbar \omega_{1}=55 \mathrm{meV}$ to $\hbar \omega_{2}=60 \mathrm{meV}$. This calculation represents the upper limit for the number of phonons that survive until the next electron arrives. With $105 \mathrm{Si}$ OM phonons per electron, we can calculate the beam-induced non-equilibrium phonon generation rate $\dot{n}_{\text {beam }}=$ $1.966 \times 10^{9} \mathrm{Si} \mathrm{OM}$ phonons per second. In addition, from the first-principles calculation, we find that the volumetric specific for optical phonons is $C_{\mathrm{p}, \mathrm{OM}}=4.53 \mathrm{e}^{5} \mathrm{~J} / \mathrm{m}^{3} / \mathrm{K}$. If further assuming the energy of those optical phonons are uniformly confined within the range of mean free path, i.e., with the volume of a cylinder of $\pi \times(10 \mathrm{~nm})^{2} \times 50 \mathrm{~nm}$ where $50 \mathrm{~nm}$ is the thickness of the slab, the effective temperature rise due to optical phonons is estimated to be $0.13 \mathrm{~K}$, which is negligible compared with room temperature $300 \mathrm{~K}$. Thus, the equilibrium part of the phonon occupation number is well described by the Bose-Einstein distribution at $300 \mathrm{~K}$.

\section{$\underline{\text { Phonon population from Boltzmann transport equation }}$}

To properly describe the nonequilibrium phonon population near an interface, the phonon Boltzmann transport equation (BTE) with source within the time relaxation approximation writes $^{40}$,

$$
\frac{\partial f_{q v}}{\partial t}+v_{q v} \cdot \nabla f_{q v}=-\frac{f_{q v}-f_{q v, 0}}{\tau_{q v}}+\dot{n}
$$

where $f_{\boldsymbol{q} v}$ is the phonon distribution function, $\boldsymbol{v}_{\boldsymbol{q} v}$ is the group velocity, $f_{\boldsymbol{q}, 0}$ is the equilibrium phonon population i.e., the Bose-Einstein distribution, $\tau_{q v}$ is the phonon lifetime and $\dot{n}$ is the phonon generation rate due to electron energy loss. Define $g_{q v}$ as the nonequilibrium part of the phonon population as: $g_{\boldsymbol{q} v}=f_{\boldsymbol{q} v}-f_{\boldsymbol{q} v, 0}$.

As discussed in above session, the temperature rise is negligible thus $f_{\boldsymbol{q} v, 0}$ is approximated to be a constant for all modes. As a result, Eq. 1 becomes, 


$$
v_{q v} \cdot \nabla g_{q v}=-\frac{g_{q v}}{\tau_{q v}}+\dot{n}
$$

We express the equation in cylindrical coordinate,

$$
\left(v_{r} \hat{r}+v_{\theta} \widehat{\theta}\right) \cdot \nabla g\left(r, \theta, v_{r}, v_{\theta}\right)=-\frac{g\left(r, \theta, v_{r}, v_{\theta}\right)}{\tau}+\dot{n}(r, \theta)
$$

where we drop the superscript and subscript for clarity. The phonon source is represented by a step function, $\dot{n}(r, \theta)=\Theta(-r)$. The radial and tangential component of velocity are $v_{r}=v_{y} \sin \theta+$ $v_{x} \cos \theta$ and the $v_{\theta}=v_{y} \cos \theta-v_{x} \sin \theta$. When the phonon source is located far from the interface, the boundary conditions of phonon BTE are $g\left(0, \theta,\left|v_{r}\right|, v_{\theta}\right)=g\left(0, \theta,-\left|v_{r}\right|, v_{\theta}\right), g$ is continuous at $r=a$ and $g\left(v \tau, \theta,-\left|v_{r}\right|, v_{\theta}\right)=0$, where $v=\sqrt{v_{x}^{2}+v_{y}^{2}}$. Thus, the solution is,

$$
g=\left\{\begin{array}{c}
\dot{n} \tau\left(1-e^{-\frac{v_{r} r+\sqrt{a^{2} v^{2}-r^{2} v_{\theta}^{2}}}{v^{2} \tau}}\right), r<a \\
2 \dot{n} \tau e^{-\frac{v_{r} r}{v^{2} \tau}} \sinh \left(\frac{\sqrt{a^{2} v^{2}-v_{\theta}^{2} r^{2}}}{v^{2} \tau}\right), r \geq a
\end{array}\right.
$$

Near the interface, the reflected phonon must be added in the solution, as explained in Extended Data Fig. 19. We first consider specular reflection with oblique and normal incidence and find only normal incidence towards interface causes enhancement in the nonequilibrium phonon population, shown in Extended Data Figs. 8a-8c. Then, we consider the case of reflected nonequilibrium phonon population from diffuse reflection, $g^{\prime \prime}=R \int g^{+}(\alpha) \mathrm{d} \alpha / \pi$, where $g^{+}(\alpha)$ is the diffusely reflected phonon population with $\alpha$ being the angle for diffusely reflected phonons. As shown in Extended Data Figs. 8d and 8e, we find the diffuse reflection does not contribute to the phonon population enhancement except extremely close to interface. In conclusion, only specular reflection of normal incident phonons causes enhancement in nonequilibrium phonon population. In Extended Data Fig. 12, we have shown the nonequilibrium phonon population only considering normal incidence. Considering the exponential decay feature of expression in Eq.3, the reflected phonon population can be expressed by,

$$
\boldsymbol{g}^{\prime}=\boldsymbol{A} \cdot \boldsymbol{S} \cdot \boldsymbol{R} \boldsymbol{e}^{-\mathrm{z} / \Lambda}
$$

when the distance to interface $z$ is larger than beam radius $a$. 
We want to emphasize that although we assume the same mean free path for all optical phonons as well as same lifetime while those properties are actually momentum-dependent, our model based on BTE qualitatively rather than quantitively captures the physics of interface scattering.

\section{The Local Phonon Density of States}

The density of states for an abrupt and a gradual interface are calculated to see if local density of states of $\mathrm{Si}$ is modified by the presence of the $\mathrm{Si} / \mathrm{Ge} \mathrm{QD}$. The local density of states is obtained from the imaginary part of green's function, defined by $D\left(\omega, \boldsymbol{R}_{I}\right)=$ $\sum_{\boldsymbol{q} \lambda} u_{\boldsymbol{q} \lambda, \alpha}^{*}\left(\boldsymbol{R}_{I}\right) u_{\boldsymbol{q} \lambda, \alpha}\left(\boldsymbol{R}_{I}\right) \delta\left(\omega-\omega_{\boldsymbol{q} \lambda}\right)$, where $u_{\boldsymbol{q} \lambda, \alpha}$ is the phonon eigenvector along $\alpha$ direction and $\boldsymbol{R}_{i}$ is the position of $I^{\text {th }}$ atom. We use tetrahedron smearing to sample the states with certain frequency ${ }^{41}$. The force constant is calculated from Stillinger-Weber (SW) potential ${ }^{42}$ with lattice constant chosen as $5.431 \AA$. A $20 \times 20 \times 18 q$ point mesh is used to calculate density of states. Our calculation shows that, the local density of states of $\mathrm{Si}$ are insensitive to the interface structure (Extended Data Fig. 6). With this, we affirm that the local density of states does not contribute to the enhancement of EELS intensity.

\section{Determination of the Reflection Coefficient by Green's Function}

To obtain the reflection coefficients for abrupt and gradual interfaces, we followed Khomyakov's mothed of using Green's function to calculate reflection matrix ${ }^{43}$. We first constructed a siliconquantum dot interface structure, where the leads are semi-infinite silicon and quantum dot along [001] direction, respectively and the central device is the silicon/quantum dot interface. The mass of the quantum dot lead region is $33.1 \mathrm{u}$, which corresponds to a virtual atom of mass equal to $0.89 m_{\mathrm{Si}}+0.11 m_{\mathrm{Ge}}$. The retarded Green's function for the device is written as, $G^{r e t}\left(\omega, \boldsymbol{k}_{\|}\right)=$ $\left(\omega^{2} I-D_{C}\left(\boldsymbol{k}_{\|}\right)-\Sigma_{L}\left(\omega, \boldsymbol{k}_{\|}\right)-\Sigma_{R}\left(\omega, \boldsymbol{k}_{\|}\right)\right)^{-1}$, where $D_{C}\left(\boldsymbol{k}_{\|}\right)$is the dynamical matrix of device with transverse momentum $\boldsymbol{k}_{\|}$. The self-energy of left and right lead is $\Sigma_{L / R}\left(\omega, \boldsymbol{k}_{\|}\right)=$ $V_{L / R} g_{L / R}^{r e t} V_{L / R}^{\dagger}$ and $g_{L / R}^{r e t}$ is the surface Green's function, which is calculated using the decimation technique and $V_{L / R}$ is the submatrix of dynamical matrix describing the coupling between the lead and the central device. The reflection matrix is $r_{L L}=\frac{2 i \omega}{a_{L}} \sqrt{V_{L}^{r e t}}\left[U_{L}^{r e t}\right]^{-1}\left(G_{L}^{r e t}-\right.$ $\left.Q_{L}^{-1}\right)\left[U_{L}^{a d v^{\dagger}}\right]^{-1} \sqrt{V_{L}^{a d v}}$, where $V_{L}^{a d v / r e t}$ is the velocity matrix, $U_{L}^{a d v / r e t}$ is the matrix of eigenvectors and $a_{L}$ is the lattice constant in transport direction. The reflection coefficient for $i$ th phonon mode in left lead is $R_{i}^{L}=\sum_{j}\left(\left|r_{L L}\right|^{2}\right)_{i j}$. We use Stillinger-Weber (SW) potential ${ }^{42}$ to calculate the dynamical matrix, which generally leads to slightly overestimated phonon frequencies of silicon. Extended Data Fig.9 shows that there is a small difference between the abrupt and gradual interfaces.

\section{Effect of Strain on the Si OM}

We also investigate if the strain can lead to the observed enhancement in EELS. The functional type for the pseudopotential is the Projector Augmented Wave ${ }^{36}$ with Perdew-Burke-Ernzerhof 
(PBE) exchange-correlation and nonlinear core correction ${ }^{38}$. The energy cutoff is 36 Ry and a $4 \times 4 \times 4$ Monkhorst-Pack mesh is used. The lattice constant at equilibrium (zero strain) is found to be $5.4662 \AA$ by minimizing the total energy. $2 \times 2 \times 2$ supercells with 64 atoms are used for calculating the harmonic force constant. The DFT calculation is conducted using Quantum ESPRESSO package ${ }^{44}$ and the phonon calculation is carried out using phonopy ${ }^{45}$ package. Through DFT calculation of phonon band and density of states of Si with different strain, we find the strain effect always modifies the phonon density of states and shifts the frequency simultaneously (Extended Data Fig. 16), whilst in experiment, we only observe intensity difference of EELS but not the phonon frequency in Si (Figs. 2b and 3a). This suggests strain effect cannot explain the experimental observation. 

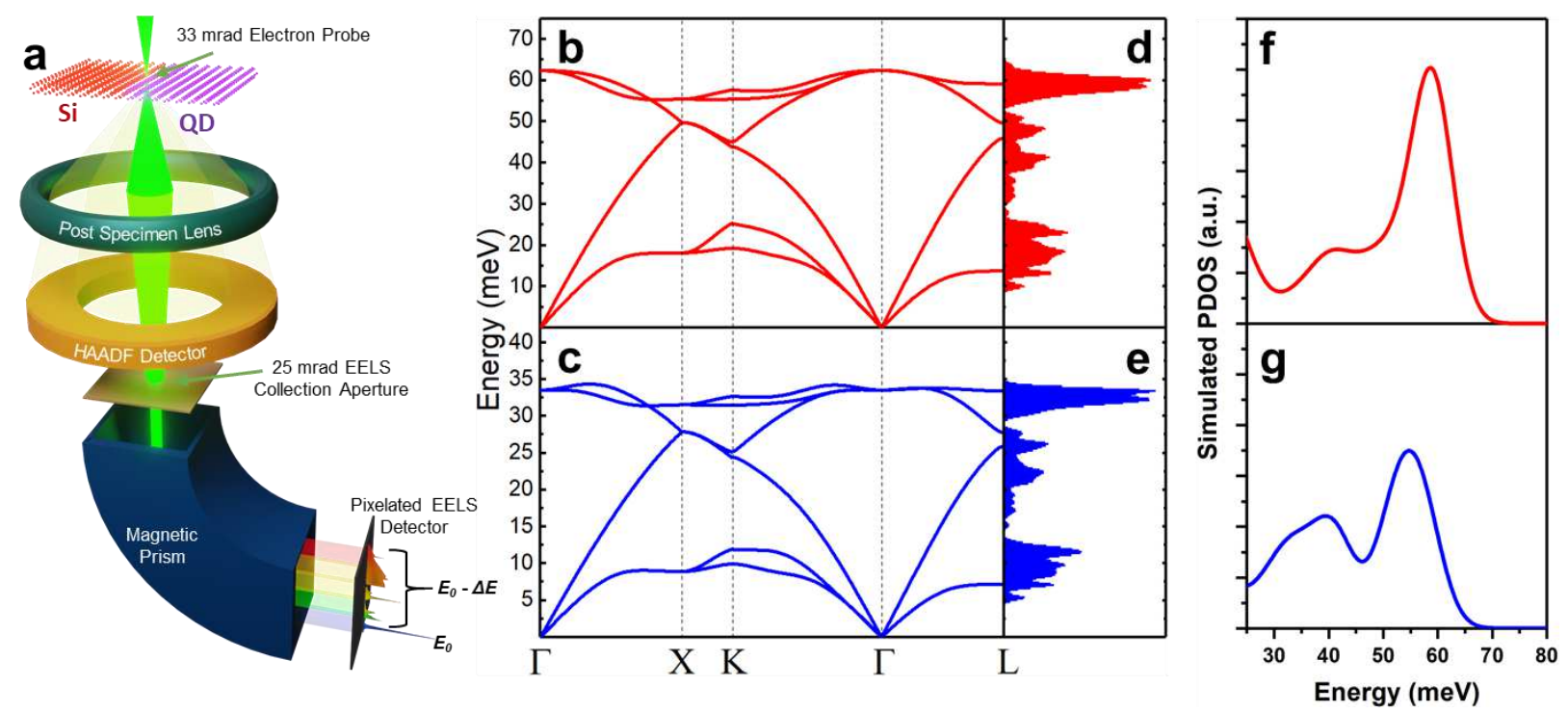

Extended Data Fig. 1 | Experimental vibrational EELS setup and phonon dispersion curves. a, Schematic of the STEM EELS experimental setup. An electron beam with a convergence semiangle of $\alpha=33 \mathrm{mrad}$ is focused to form a $1.5 \AA$ wide probe on the sample. Electrons are then elastically and inelastically scattered by the sample. High angle scattered electrons are recorded by the HAADF detector. The lower angle scattered electrons enter the spectrometer with a collection semi-angle of $\beta=25 \mathrm{mrad}$ and are dispersed based on energy. Elastically scattering electrons form the ZLP while inelastic electrons contribute to the vibrational signal. b, c, Phonon dispersion relations of bulk $\mathrm{Si}$ and $\mathrm{Ge}$, respectively. $\mathbf{d}$, e, Phonon density of states of bulk Si and $\mathrm{Ge}$, respectively. f, g, Optical portion of the calculated phonon density of states in Si and SiGe QD respectively after convolution with a gaussian of width $7 \mathrm{meV}$. 

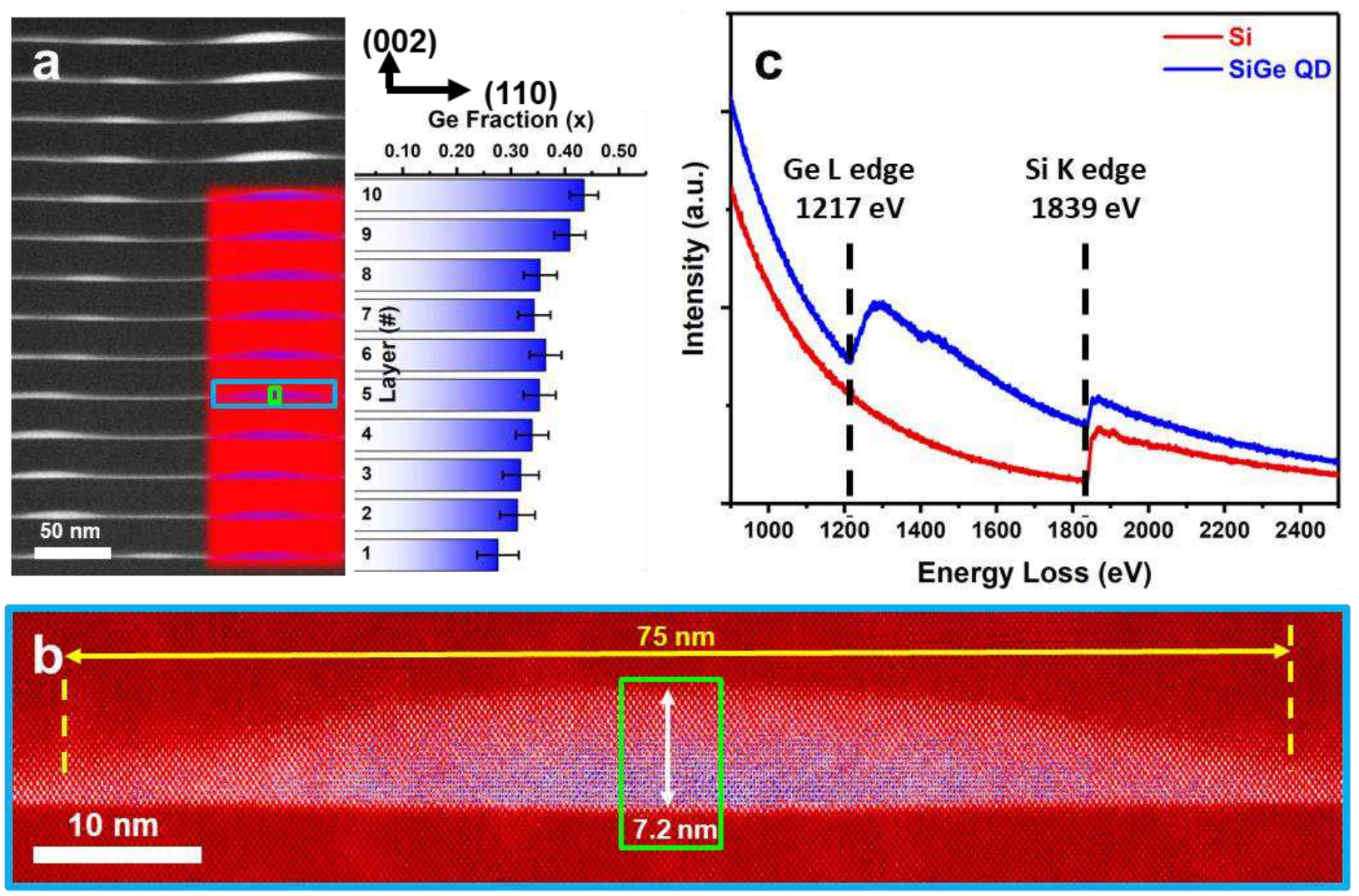

Extended Data Fig. 2 | STEM characterization of quantum dot layers. a, Low magnification STEM image of Si-Ge quantum dot (QD) superlattice. Due to the Z-contrast property of HAADF imaging, the bright regions are $\mathrm{SiGe}$, while the darker regions are interlayer Si. The crystal orientations are indicated as [001] for the growth direction and [110] for the lateral, planar direction. A high-angle annular dark-field (HAADF) image of the first 10 layers from the $\mathrm{Si}$ substrate is superimposed with an elemental EELS map. Red and blue indicate silicon and germanium concentration, respectively. Histogram plot to the right displays Ge composition fraction of the corresponding 10 layers. b, HAADF image of a single QD from the blue region in a showing dome-like structure and dislocation-free interfaces. The green box denotes the region in Fig. 1a. c, Core-loss EEL spectra of interlayer Si and SiGe QD. Core-loss spectrum in interlayer Si shows only 1 peak corresponding to the Si $K$ edge while the spectrum in the SiGe QD shows 2 peaks corresponding to Ge $L$ edge and Si $K$ edge. 

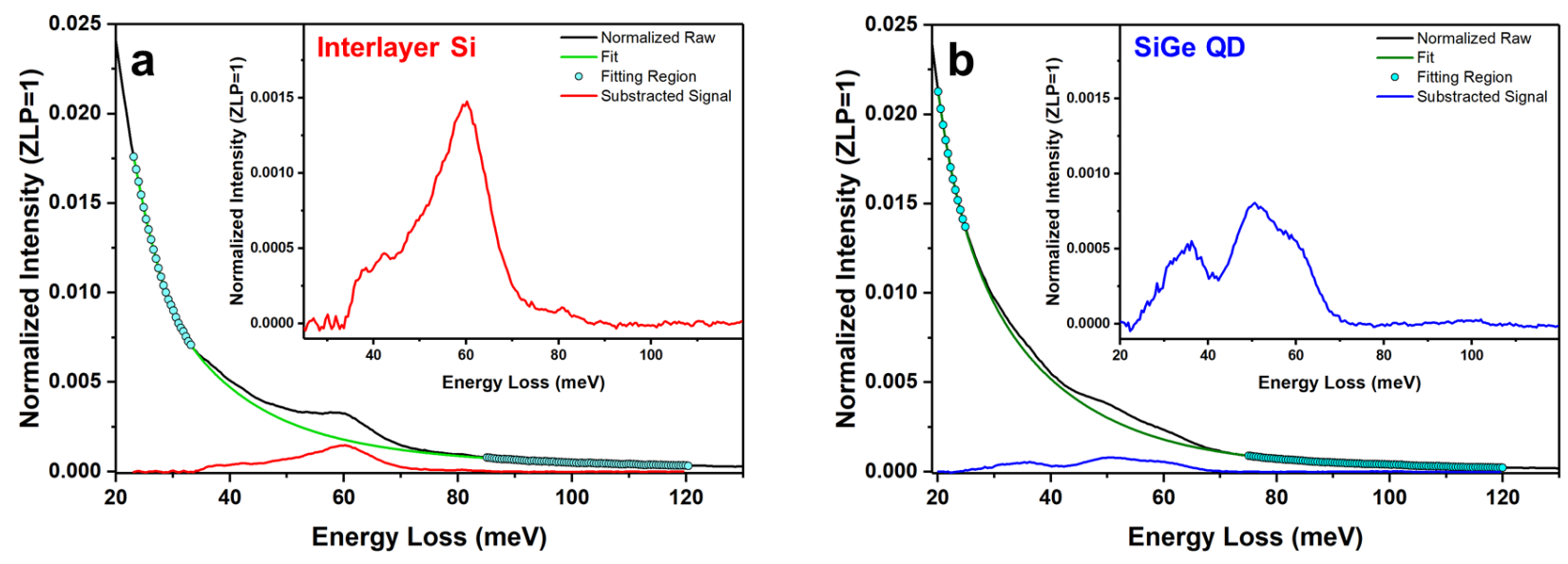

Extended Data Fig. 3 | Background subtraction of low-loss EEL spectra. Typical background subtraction procedure of spectra acquired in interlayer Si a, and SiGe QD b, respectively. The green colored line represents the normalized as-acquired spectra in the corresponding regions. Cyan dots represent the spectrum region used for fitting the background. The red and blue curves in the main plot and in the inset represent the background subtracted signal respectively in a and b. 


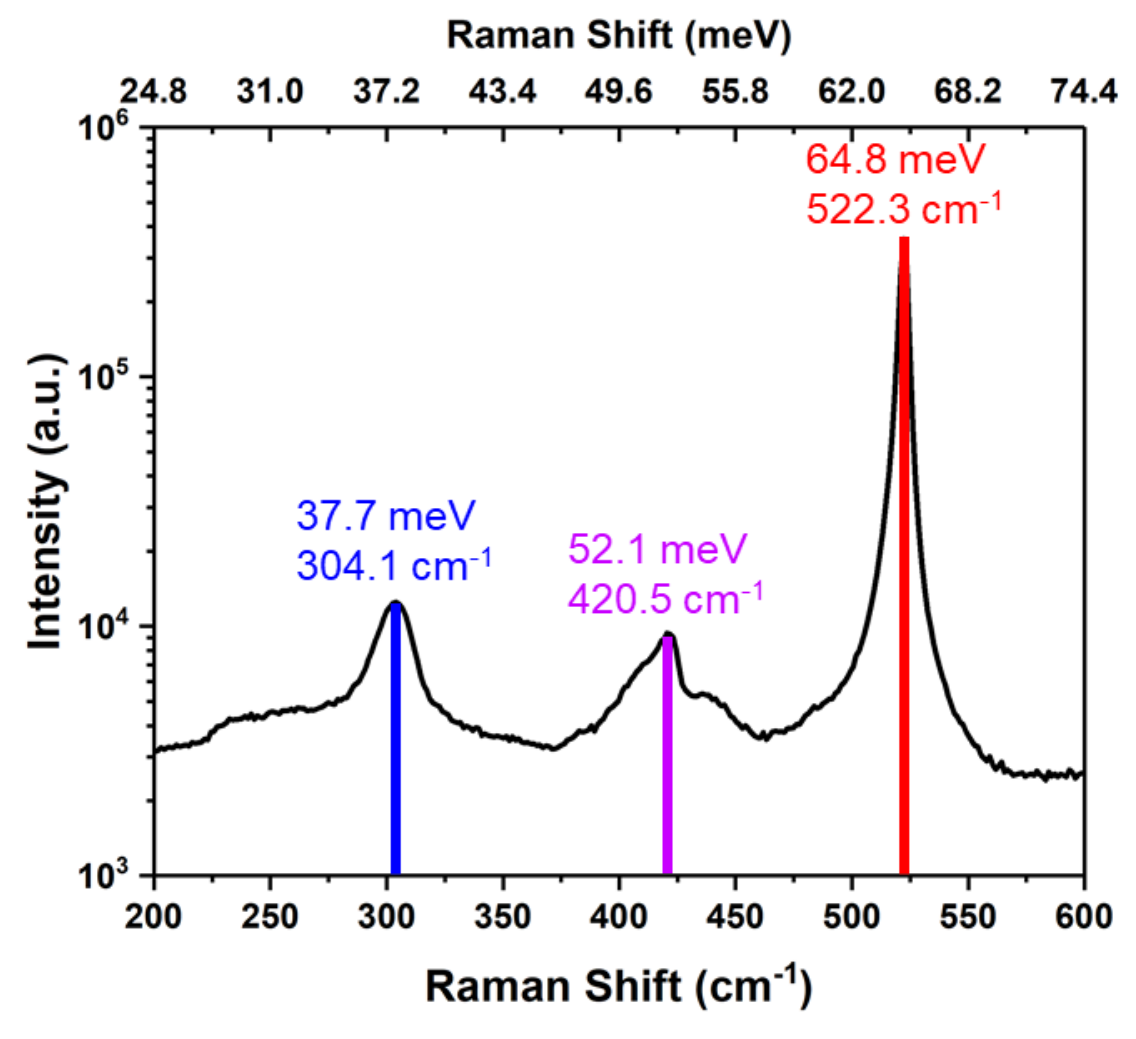

Extended Data Fig. 4 | Raman spectrum. Log spectrum from SiGe QD superlattice sample grown on silicon. The spectrum shows 3 distinct peaks at 37.7, 52.1, and $64.8 \mathrm{meV}$ arising from $\mathrm{Ge}, \mathrm{SiGe}$, and Si optical modes, respectively. Raman spectroscopy only offers sample-averaged information of lattice vibrations. 

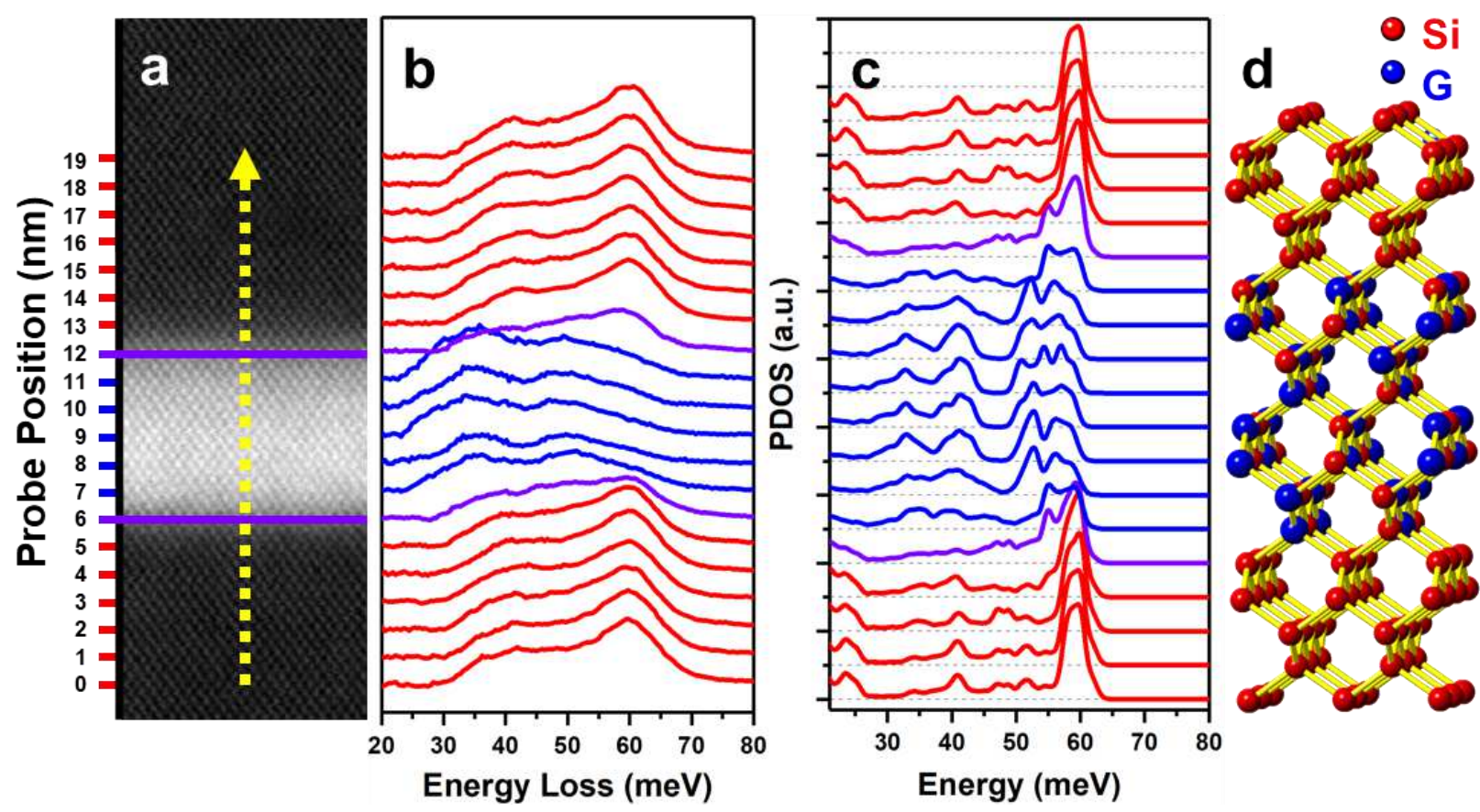

Extended Data Fig. 5 | Line scan across QD. a, HAADF image of the region consisting of top and bottom interfaces of a single QD. The yellow arrow notes the direction of the line scan and the purple lines mark the QD interfaces. b, Stacked background subtracted spectra with red, blue, and purple curves representing phonon signals in interlayer $\mathrm{Si}, \mathrm{SiGe} \mathrm{QD}$, and interface signals. Stacked positions correspond to the probe positions labeled in a. c, Stacked calculated phonon density of states and $\mathbf{d}$, associated atomic structure. 

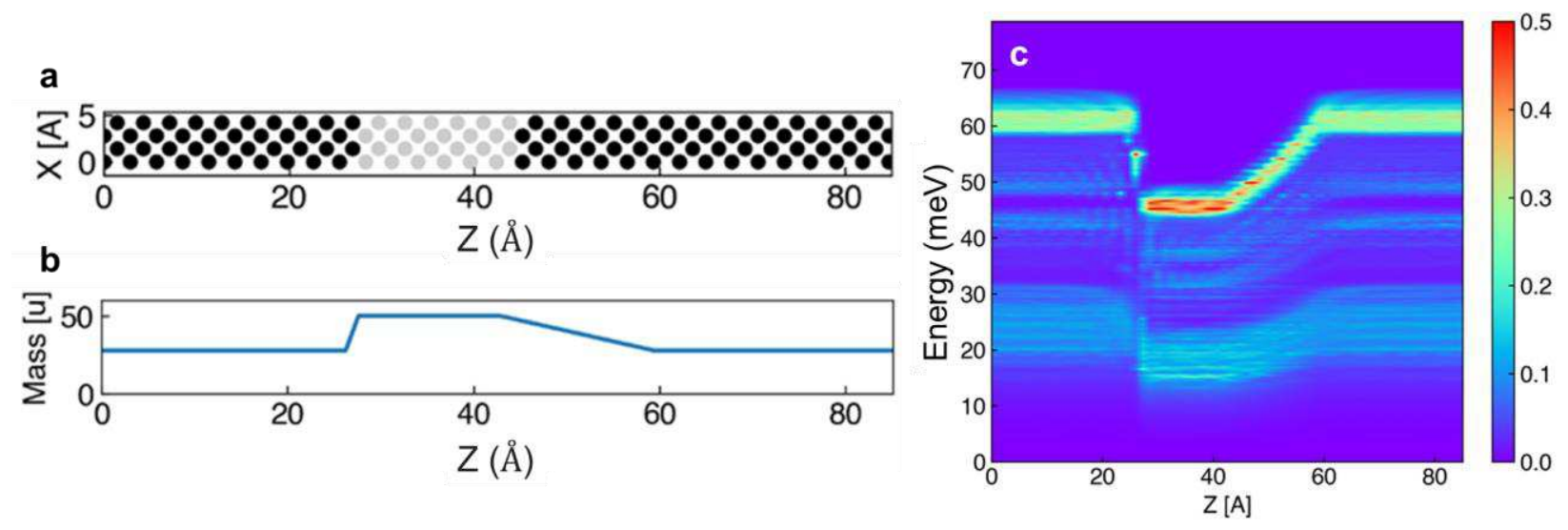

Extended Data Fig. 6 | Local Phonon Density of States of a SiGe nanostructure. a, Superlattice supercell with SiGe alloy structure formed by atoms representing the average mass of $\mathrm{Si}$ and $\mathrm{Ge}$ with the gray color indicating an atomic mass of $\mathrm{Si}_{0.5} \mathrm{Ge}_{0.5}$. The 12 layers of atoms to the right of the gray colored ones have a mass gradient that transitions from $\mathrm{Si}_{0.5} \mathrm{Ge}_{0.5}$ to pure $\mathrm{Si}$. b, Mass profile of a along the $z$-direction. Near the abrupt Si-QD interface, the mass abruptly changes. The gradual interface is modeled by continuously changing the mass profile as a function of position. c, The local density of phonons for vibrational modes along the $z$-direction in the superlattice depicted in $\mathbf{a}$. 


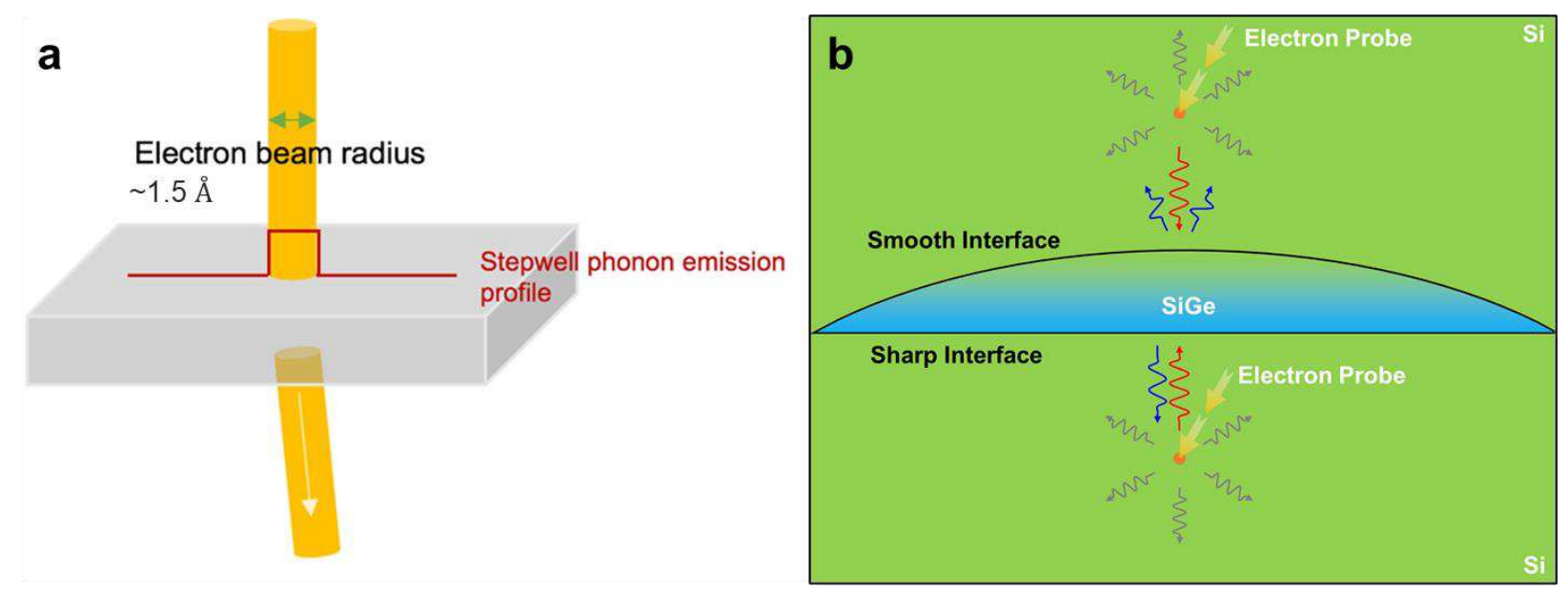

Extended Data Fig. 7 | Phonon generation by the electron beam. a, Schematic for the electron beam as a phonon emission source in EELS. b. Two-dimensional geometry from top view. The blue region indicates the quantum dot. The beam is indicated by the orange circle and its area is exaggerated for visual convenience. The curvature is measured to be about $35^{\circ}$ with the radius around $1520 \AA$. The mean free path of $\mathrm{Si}$ OM phonons is chosen to be $25 \mathrm{~nm}$ with a group velocity of $1000 \mathrm{~m} / \mathrm{s}$. 
a

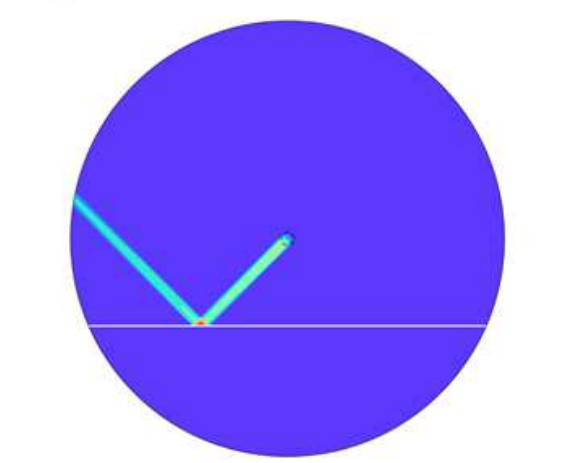

b

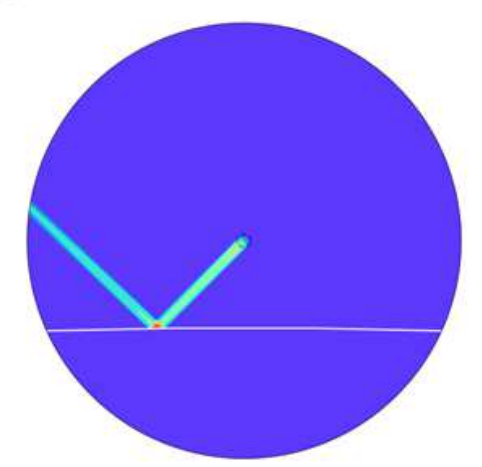

C

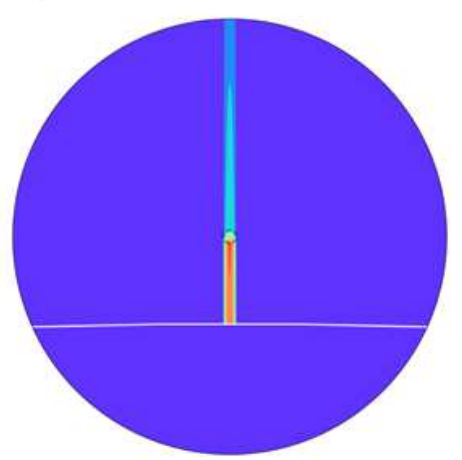

d

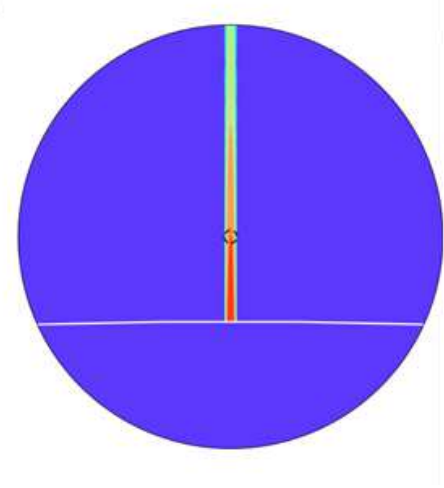

e

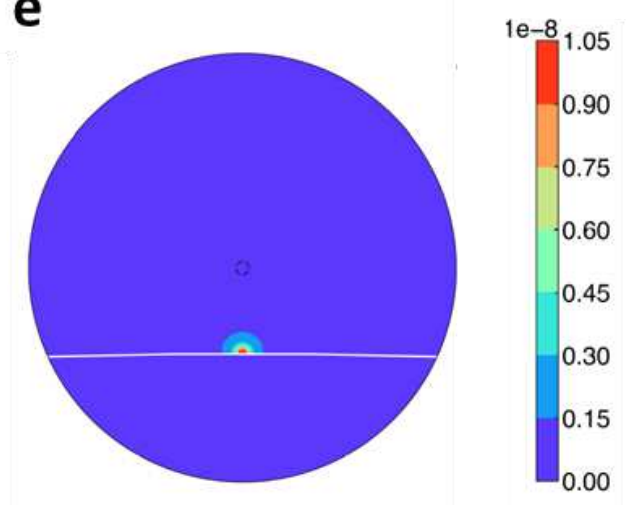

Extended Data Fig. 8 |Effect of interface geometry and specularity on phonon reflection. ac, The non-equilibrium phonon distribution function $g$ for a phonon mode with group velocity along certain polar angle that consists of the incoming and corresponding fully specularly reflected phonons $(S=1, R=1)$ near a flat $\mathbf{a}$ and curved $\mathbf{b}$, interface with source phonon moving with along $\theta=-135^{\circ}$ and a curved interface $\mathbf{c}$, with source phonon moving along $\theta=-90^{\circ}$, respectively. We find that only normal incident phonons have significant enhancement in the nonequilibrium phonon population. $\mathbf{d}, \mathbf{e}$, The fully specularly reflected $(\mathrm{S}=1, \mathrm{R}=1)$ and diffusively scattered $(\mathrm{S}=0, \mathrm{R}=1)$ nonequilibrium phonon distribution $g^{\prime}\left(r^{\prime}, v_{r}^{\prime}, v_{\theta}^{\prime}\right)$ and $g^{\prime \prime}(r)$ respectively, when the incoming phonon at normal to the interface. Only reflected phonon population are plotted for $\mathbf{d}$ and $\mathbf{e}$. From $\mathbf{e}$, we find the diffusively scattered part is highly localized near the interface such that we can neglect the enhancement of phonon population from the contribution of diffusively scattered phonons. The small, dashed circles in the center of a-e indicate the location of the phonon emission source. The color bar represents the phonon population with arbitrary units. 

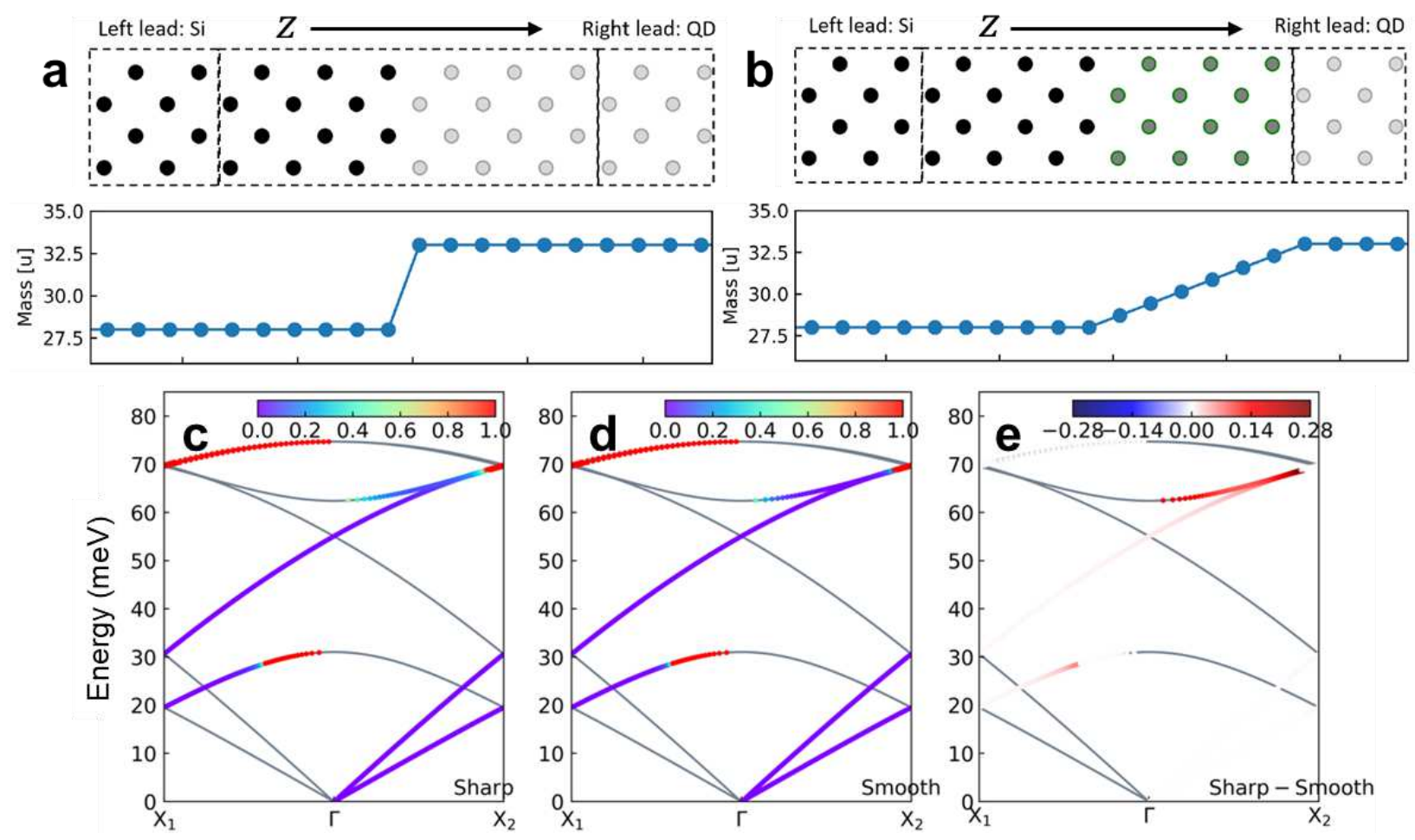

Extended Data Fig. 9| The reflection coefficients for phonon states of silicon through [001] direction. a, b, Atomic structure (upper panel) and corresponding mass profile (lower panel) of abrupt Si-QD interface $\mathbf{a}$ and gradual Si-QD interface $\mathbf{b}$. In the atomic structures, the darker the atom's color is, the lighter the atomic mass is. For an atomic layer with the same $\mathrm{z}$ position, the mass is the same. Periodic boundary conditions in transverse directions are used. c-e, Reflection coefficients of abrupt Si-QD interface $\mathbf{c}$, gradual Si-QD interface $\mathbf{d}$ and their difference $\mathbf{e}$ along [001] direction in reciprocal space. The transport direction normal to the interface is along $\mathrm{z}$ direction. The high symmetry point $X_{1}$ refers to $(0,0,-1) \frac{\pi}{a}$ and point $X_{2}$ refers to $(0,0,1) \frac{\pi}{a}$ in the first Brillouin zone of the 4-atom unit cell where $\mathrm{z}$ direction is [001] direction. The modal reflection coefficients are calculated from atomic Green's function. 

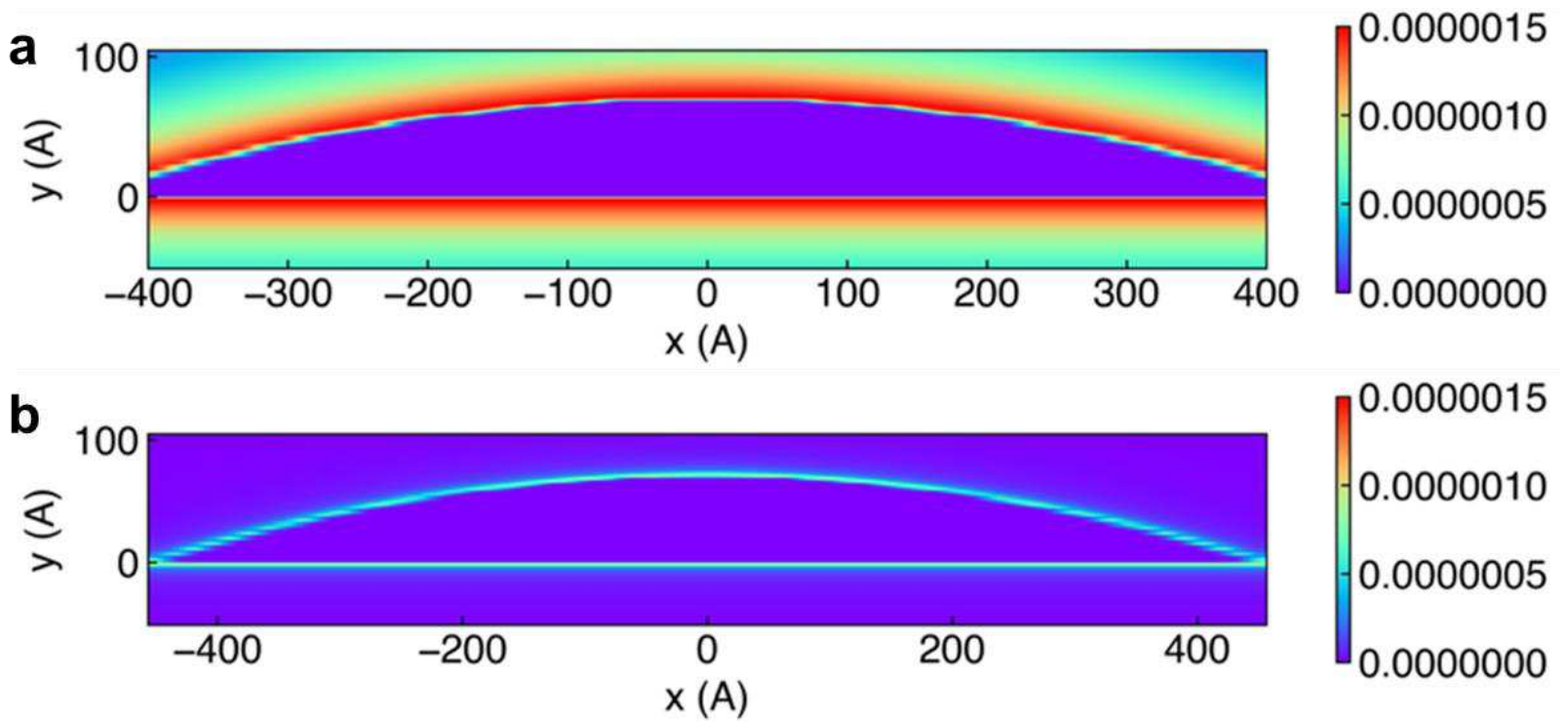

Extended Data Fig. 10 | Interface specularity vs. geometry. a, Spatially integrated distribution function of specularly reflected phonons with directions of incoming phonon velocity normal to and away from interface. Here, we assume $S=R=1$ for both abrupt and gradual interface. Since $S R$ is identical for abrupt and gradual interfaces, the reflected phonon follows a similar decay trend. In practice, the $S R$ are different for two interfaces, causing asymmetry in the phonon distribution function. $\mathbf{b}$, Diffusively reflected phonon distribution function shows strong localized feature near the surface of quantum dot, assuming $S=0$ for both interfaces. 

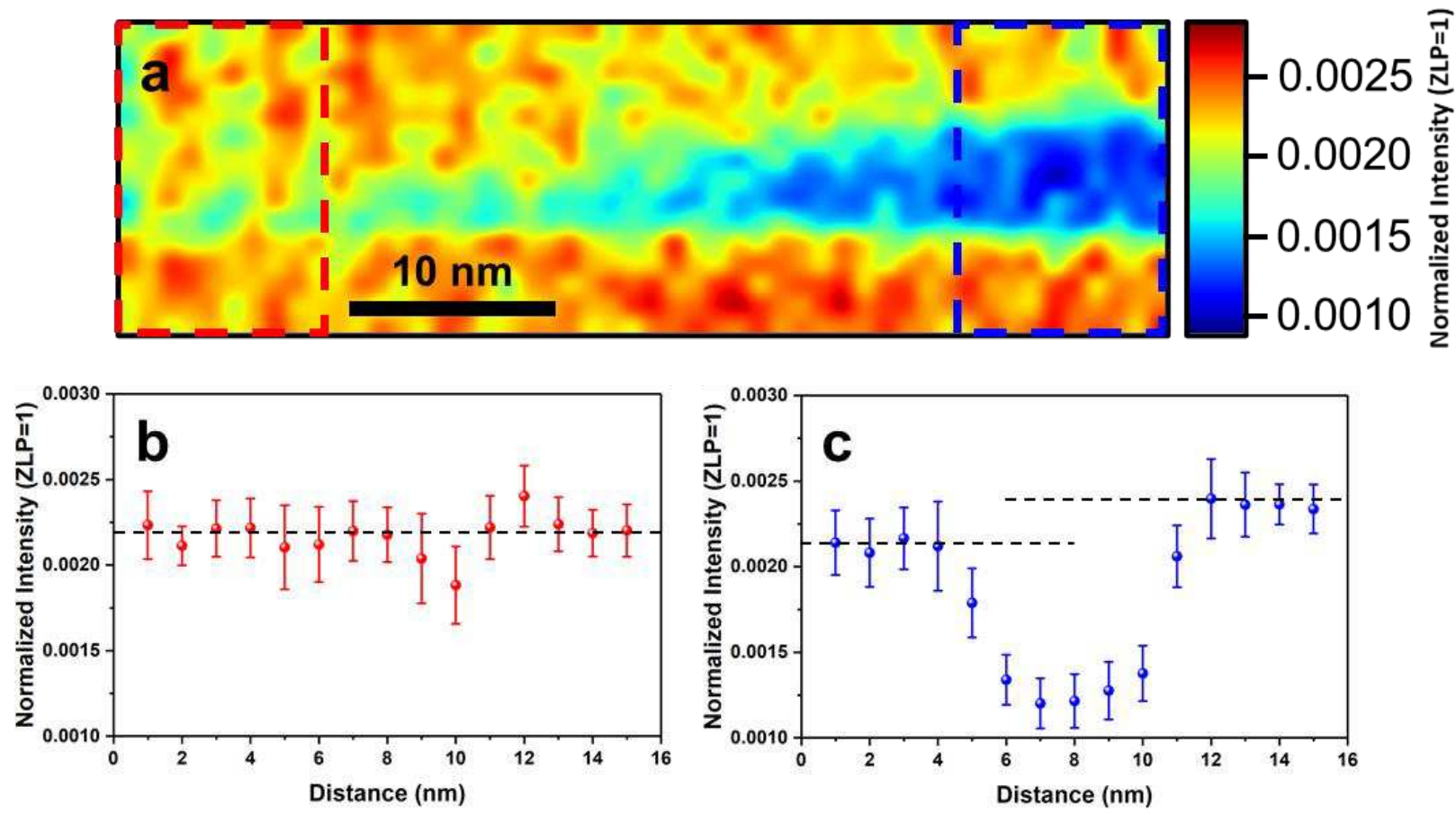

Extended Data Fig. 11 | Phonon map of QD side. a, Spatial mapping of Si phonon peak intensity of the side of a QD. b. Integrated line profile of the left-hand side of the map (red) showing consistent intensity above and below the QD edge. c, Integrated line profile of the righthand side of the map (blue) showing an intensity enhancement above and below the QD. The horizontal dashed lines in $\mathbf{b}$ and $\mathbf{c}$ show the intensity enhancement in the middle QD region and consistent intensity in the side region. 
a

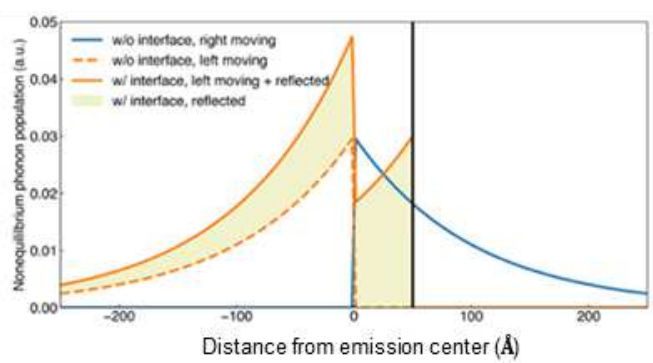

b

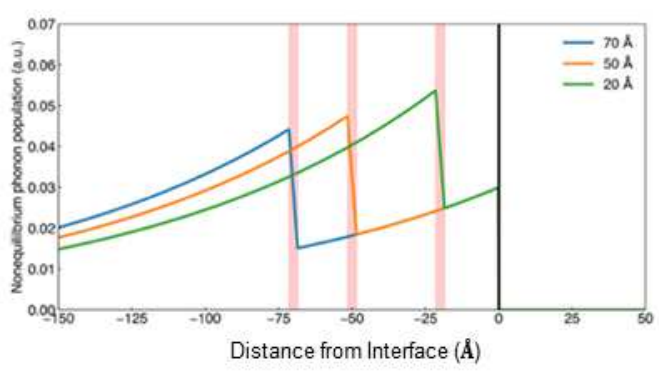

C

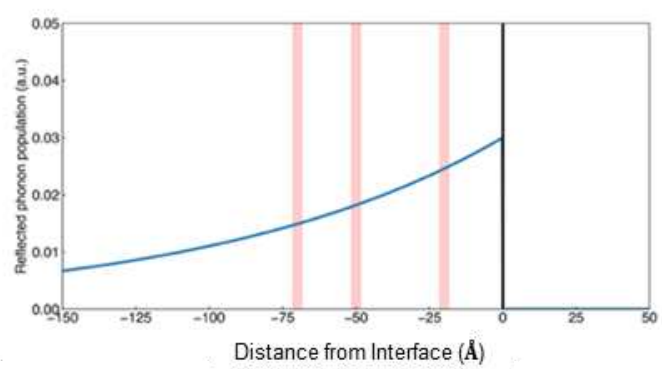

d

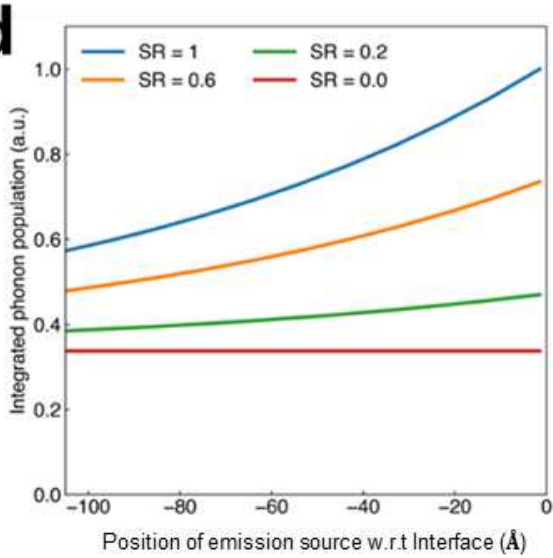

e

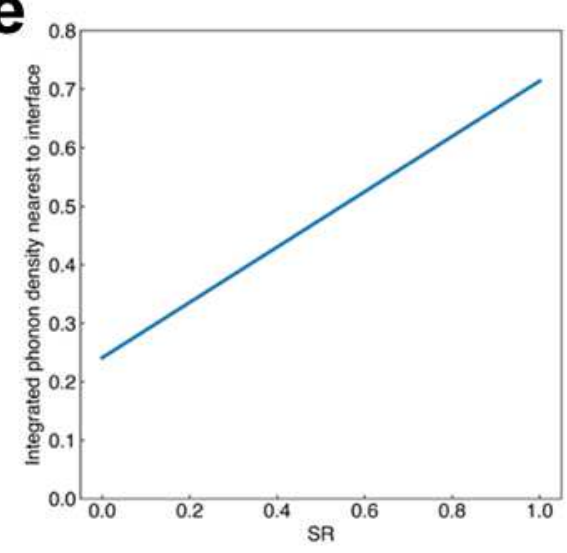

Extended Data Fig. 12 | Effect of interface specularity on phonon decay. a, The nonequilibrium phonon distribution function for phonons moving towards interface, phonons moving away from interface, and phonons that are reflected by the interface with flipped velocity direction. Zero point of position is the center of the phonon emission source and black dashed line indicates the location of interface. The blue curve describes the phonon distribution function when there is no interface. The light-yellow filled region describes the reflected phonons' contribution to phonon distribution, which only exist in the proximity of interface. The orange dashed line is the phonon distribution when including the contribution of reflected phonons. $\mathbf{b}$, When moving the phonon emission source (marked by red bar), the phonon distribution function has a different profile. Three cases with different distances to interface is plotted. With longer distance away from interface, the peak of the distribution lowers, due to smaller contributions from reflected phonons. c, The reflected phonon distribution has an exponential decay profile away from interface and changing the beam position will probe different amount of reflected nonequilibrium phonon. d, The integrated phonon population for different distance between phonon emission source and interface when different parameter $S R$ is chosen. e, The integrated phonon population at the position closest to the interface as a function of parameter $S R$. The integration is conducted within the radius of $a$. 

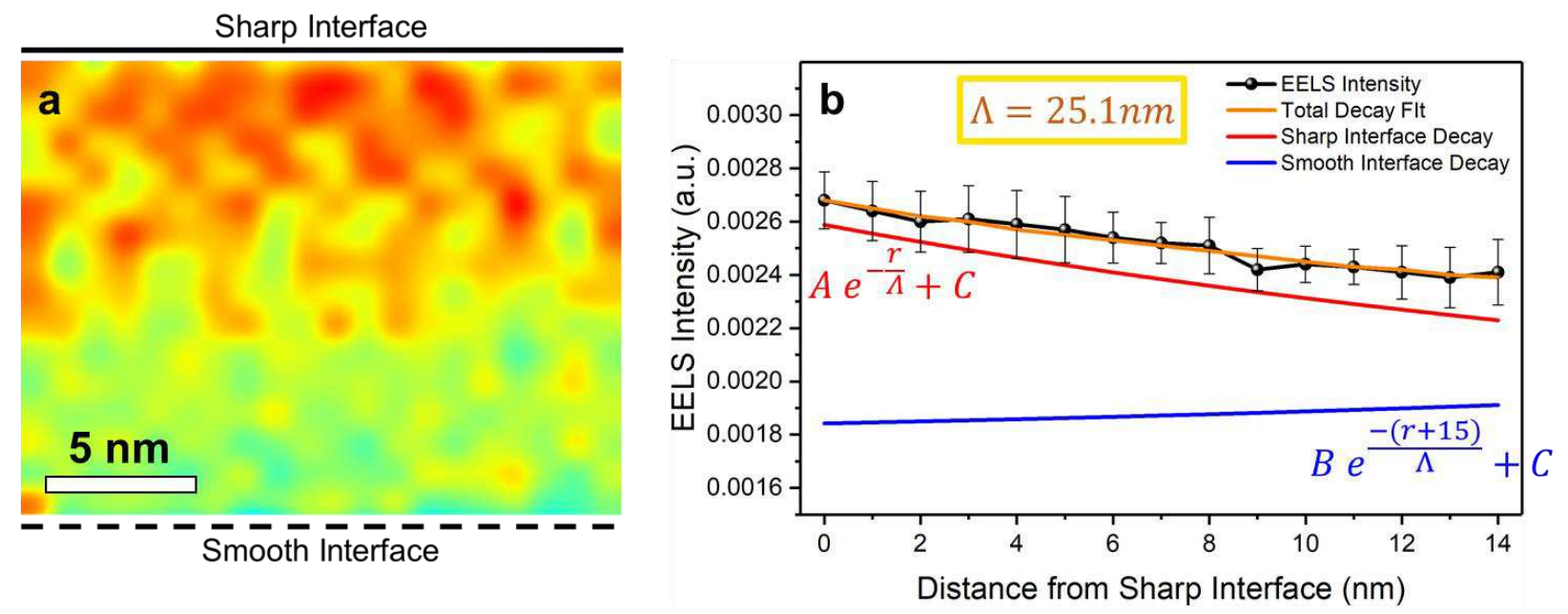

Extended Data Fig. 13 | Interlayer Si Phonon Map. a, A 20×15 nm map of Si phonon peak intensity in the interlayer Si region bounded by two QDs. b. Integrated 1D line profile of $\mathbf{a}$ with exponential decay fitting. Red and blue curves represent decay of phonon population as a function of distance from abrupt and gradual interfaces, respectively. The constants $A$ and $B$ represent the reflection induced phonon population's contribution to EELS intensity at the edge of abrupt and gradual interfaces, respectively. The constant $C$ represents the equilibrium phonon population's contribution to EELS intensity following the Bose-Einstein distribution. The orange curve is the sum of the red and blue curves. Parameters $A$ and $B$ were fit to the data and represent the reflection intensity in the immediate vicinity of the interface while $C$ was taken to represent the equilibrium phonon population that the non-equilibrium population would eventually decay to. 

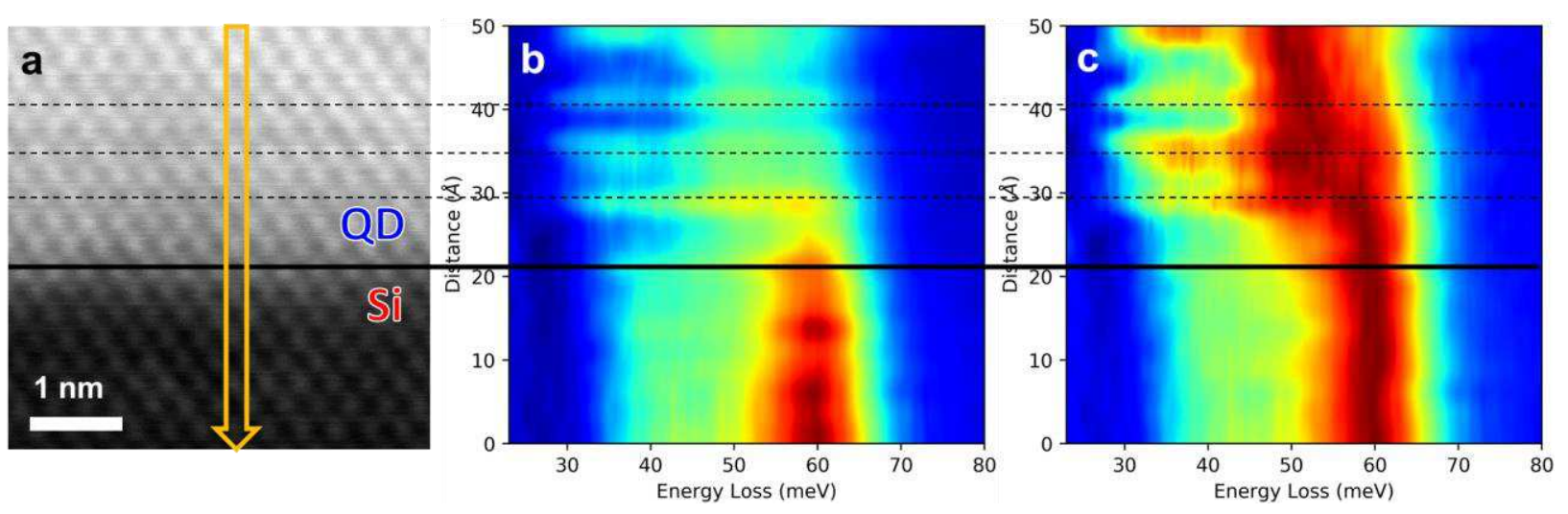

Extended Data Fig. 14 | Atomically resolved linescan across bottom abrupt QD interface. a, Atomic resolution HAADF image of an abrupt QD interface. The downward yellow arrow indicates the direction of the phonon linescan. Solid lines mark the atomic positions in the HAADF image while the dashed line marks the Si-QD interface. $\mathbf{b}$, Contour plot of linescan spectra normalized to the ZLP. Fringes are clearly seen and correlate with the atomic positions in the HAADF image in a. c, Spectral contour plot normalized to the signal maximum. Fringes are only seen on the Ge optical mode while the intensity on the Si optical mode is uniform. 

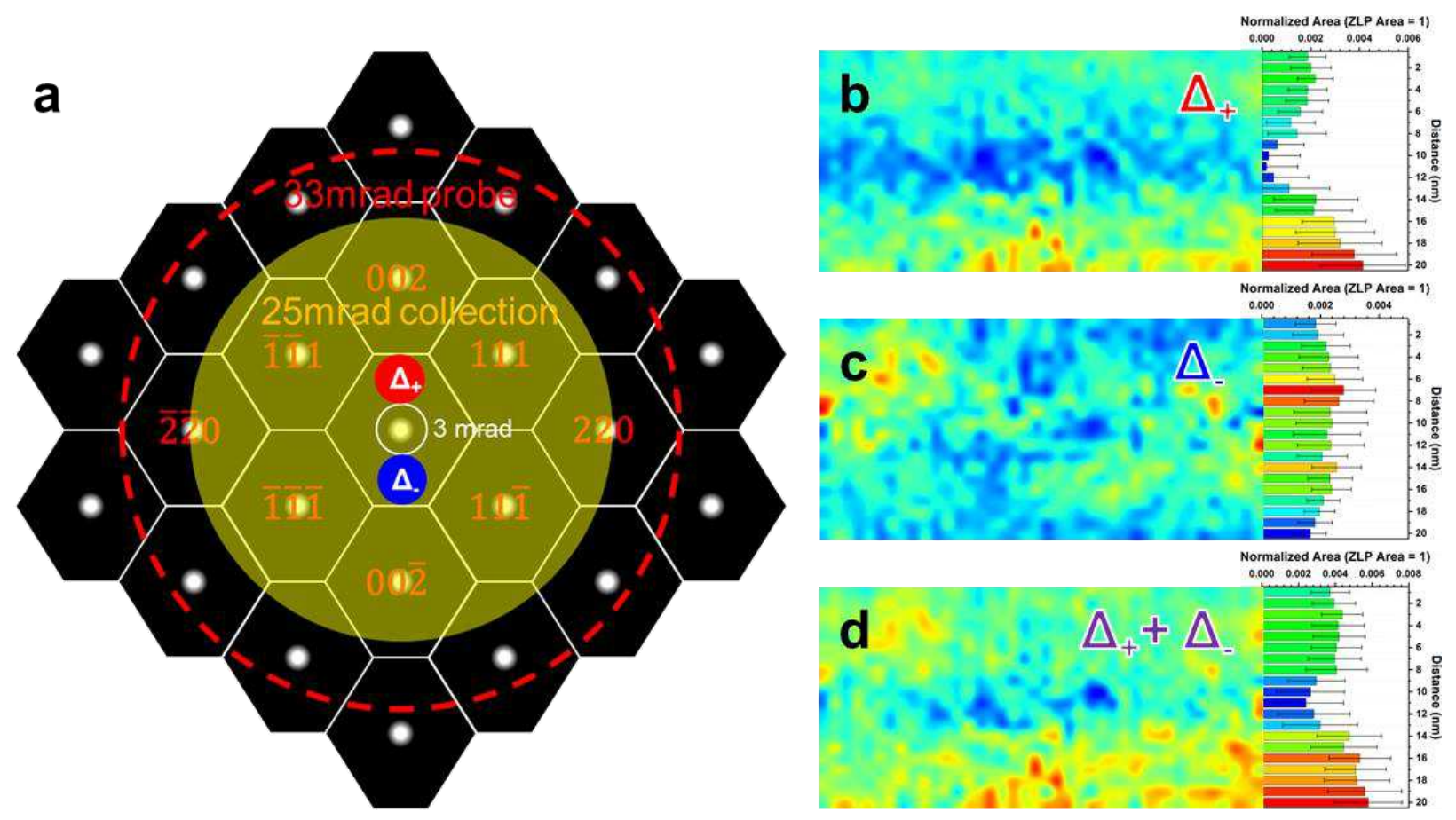

Extended Data Fig. 15 | Momentum resolved experimental beam geometry. a, Reciprocal space schematic diagram of Si. White borders denote individual Brillion zones (BZ) with the BZ's near the center labeled. White spots at the center of each BZ depict areas of dominant elastically scattered electrons while the black background is dominated by inelastically scattered electrons. Various beam-detector geometries corresponding to momentum-averaged and momentum-resolved conditions are denoted by circles with their size in semi-angle labeled. Red and blue regions in the FBZ correspond to the areas where momentum resolved differential data was obtained. b, c, Mapping of integrated background subtracted spectral intensity in the 55-65 meV region acquired from electrons deflected towards the $002\left(\Delta_{+}\right)$and $00 \overline{2}\left(\Delta_{-}\right)$crystal direction corresponding to the red and blue regions in a, respectively. $\mathbf{d}$, Combined spectral

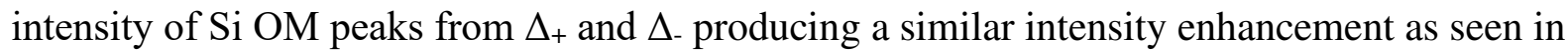
Fig. $3 \mathrm{a}$ of the main text. Phonon momenta are taken to be in the opposite direction of electron deflection. 

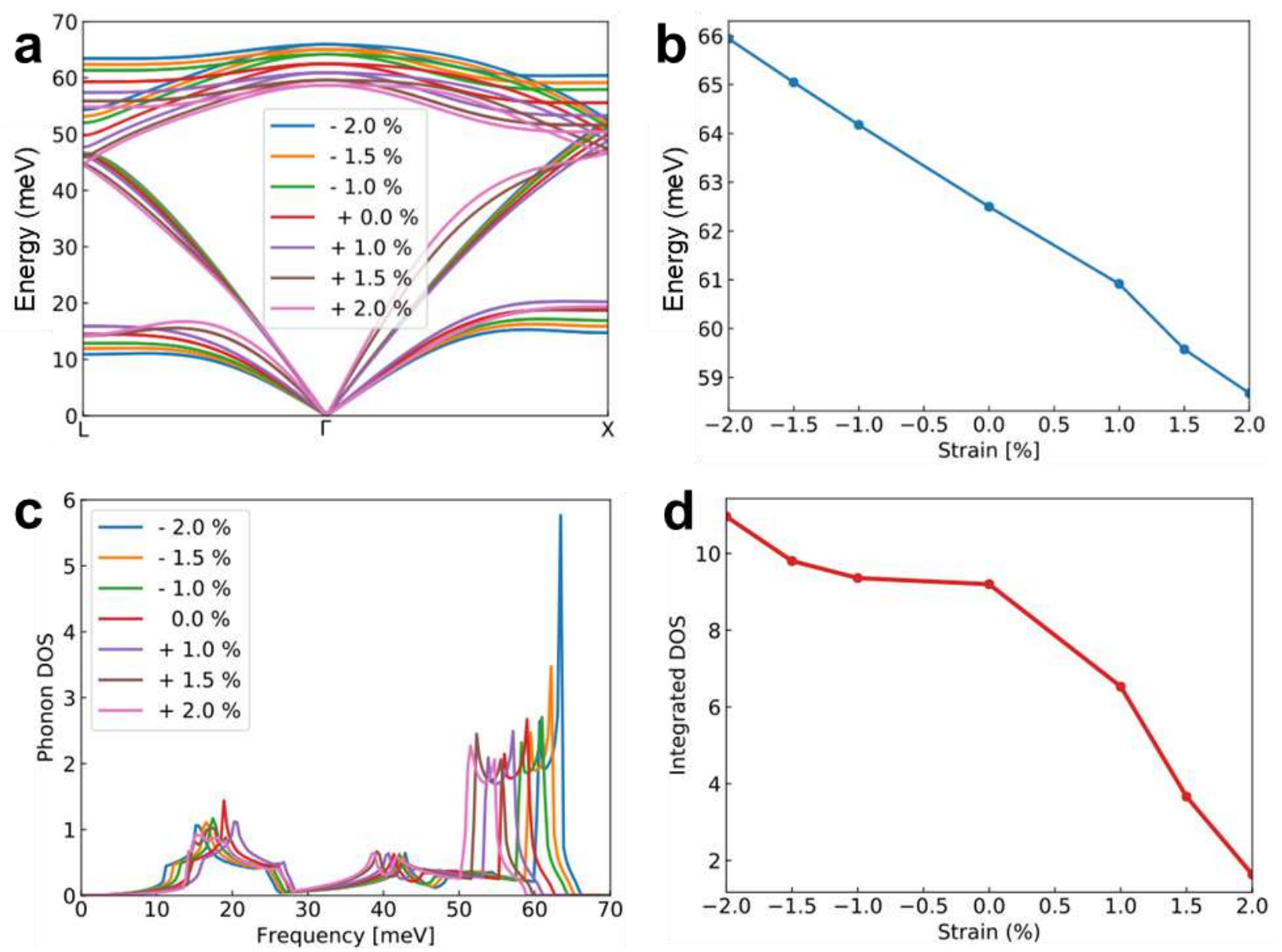

Extended Data Fig. 16 | Strain effect on Phonon Dispersion and Density of States. a, Phonon dispersion for silicon under different strains. $\mathbf{b}$, The energy of longitudinal phonon at $\Gamma$ point as a function of strain. $\mathbf{c}$, Phonon density states from DFT calculations for different strains. The percentage marks the relative change in lattice constant compared to equilibrium state. $\mathbf{d}$, The integrated phonon density of states from $55 \mathrm{meV}$ to $65 \mathrm{meV}$. As the lattice constant enlarges, the integrated DOS in the energy window from $55 \mathrm{meV}$ to $65 \mathrm{meV}$ decreases due to the red shift and broadening of optical phonons. 

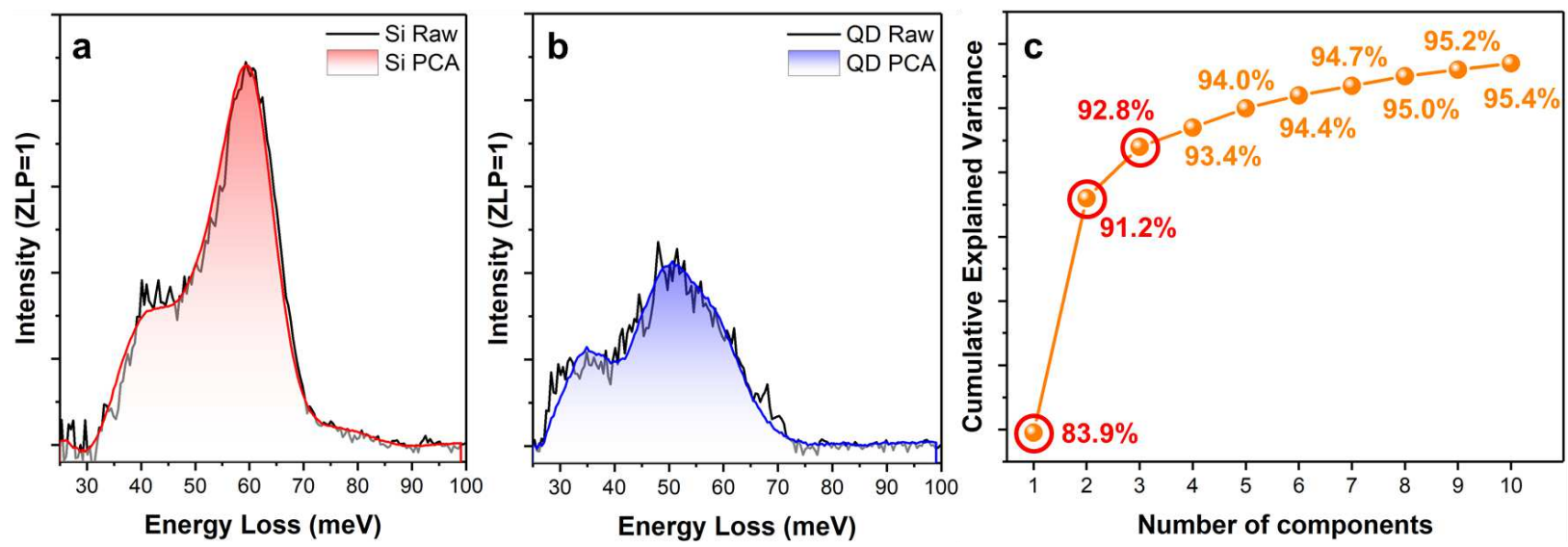

Extended Data Fig. 17 | Principal Component Analysis of Background-Subtracted Spectra. $\mathbf{a}, \mathbf{b}$, Raw background subtracted spectra in Si and QD plotted with PCA reconstructed data, respectively. c, Scree plot of cumulative explained variance. 10 eigenvector decomposition diminishing explained variance after the first 3 eigen vectors. Therefore, the first 3 eigenvectors were chosen for the decomposition giving a cumulative explained variance of $92.8 \%$. PCA reconstructed plots in $\mathbf{a}$ and $\mathbf{b}$ show that 3 eigenvectors sufficiently smoothen the data without any loss of features. 


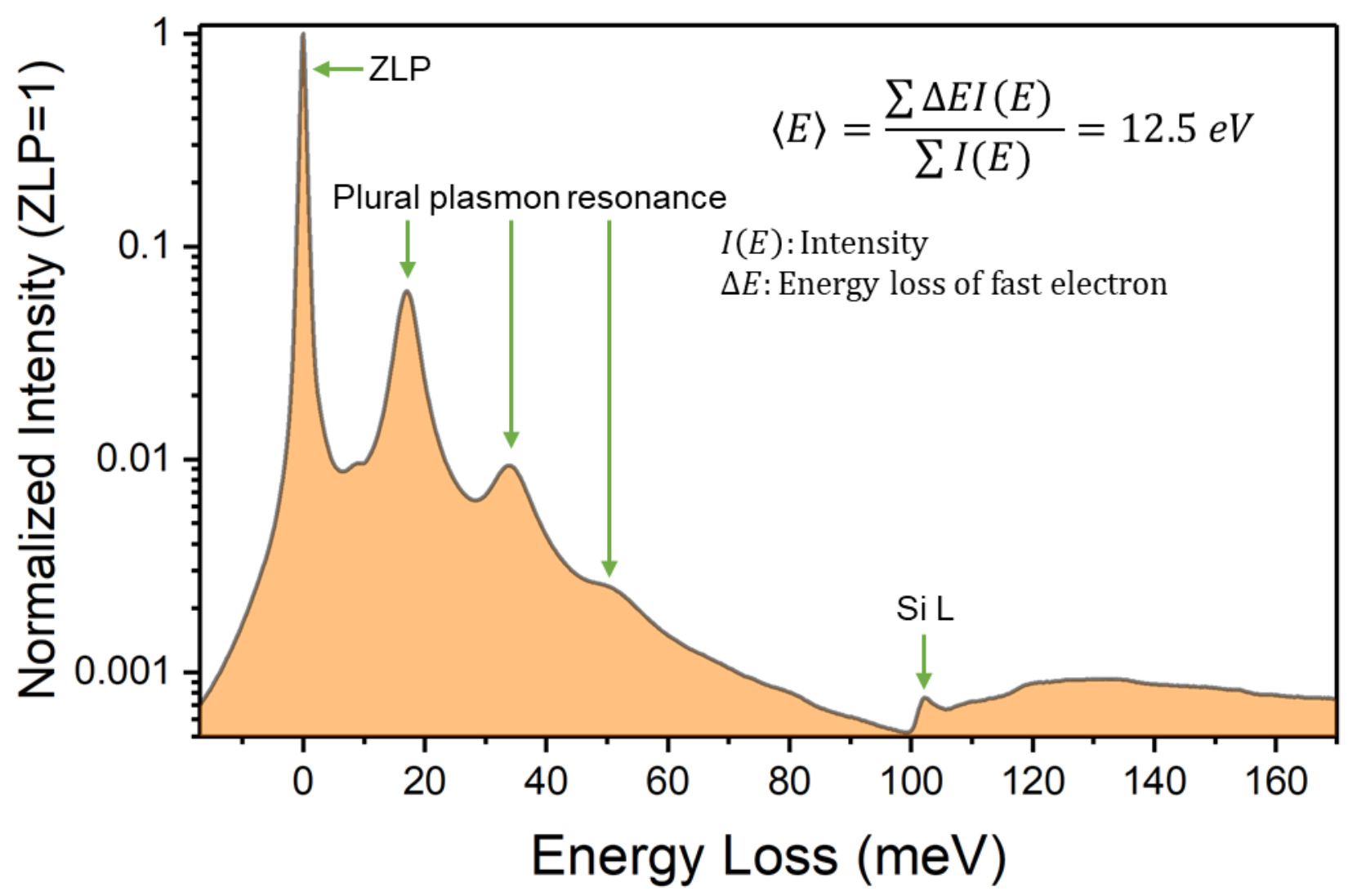

Extended Data Fig. 18 | Low-loss EELS for average energy loss on interlayer Si. An EEL spectrum contains a probability-like distribution of the energy loss of single electron. This lowloss spectrum shows plural plasmon resonance and $\mathrm{Si}$ L edge core loss that eventually decays into phonons. Weighting by Si DOS, there are 105 phonons generated per fast electron. 


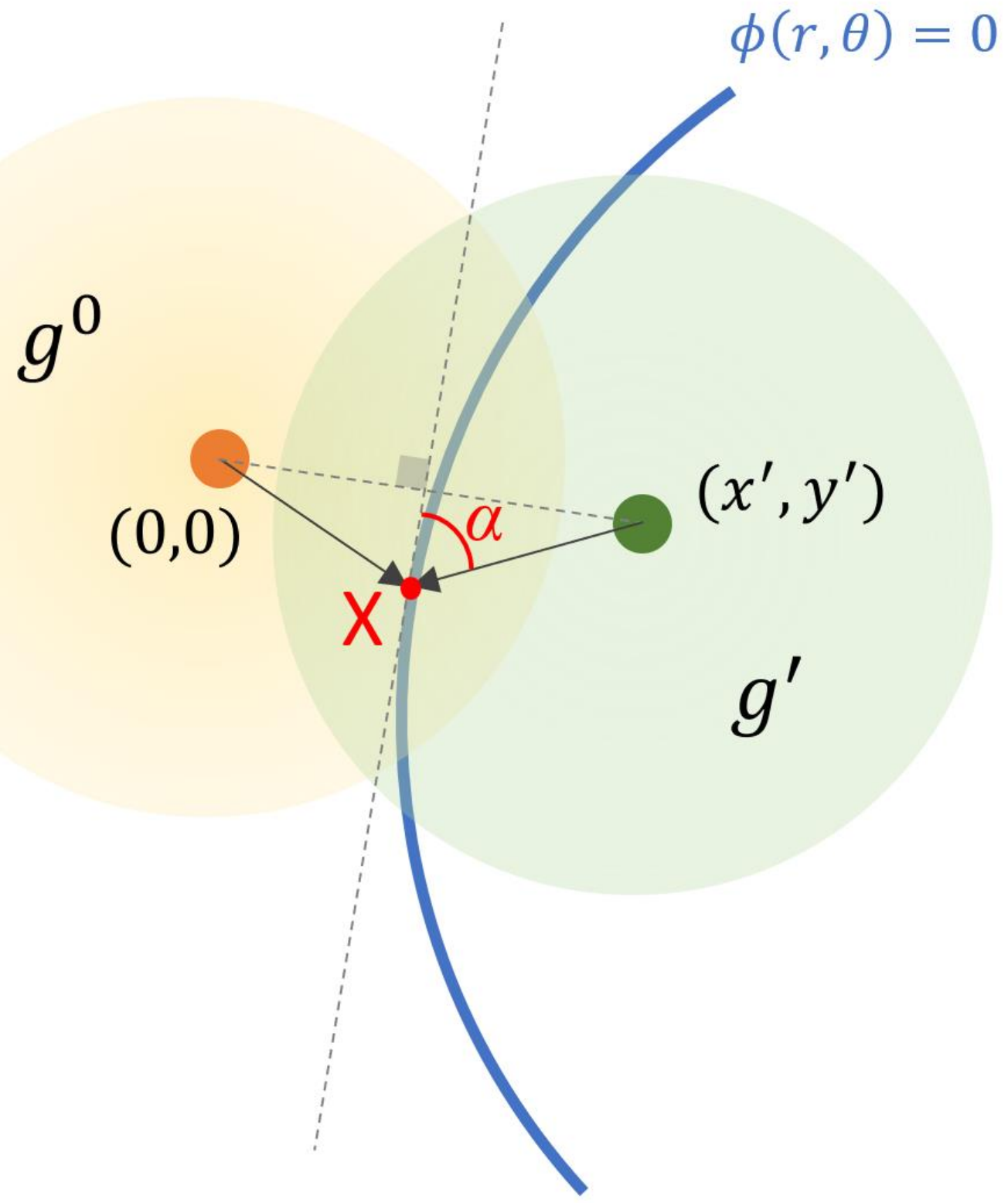

Extended Data Fig. 19 | Incoming and reflected phonon population. The blue curve represents the interface profile. The yellow region denotes the incoming phonon population $g$ given by Eq. 3 and the origin is at $(0,0)$. The reflected phonon $g^{\prime}$ is given by a similar expression with shifted origin at $\left(x^{\prime}, y^{\prime}\right)$. The arrows denote the velocity direction of incident and reflected phonons. The shifted origin for reflected phonons and the origin for incoming phonons are mirror-symmetric with respect the tangential plane at interface. 